\title{
Penrose limits in non-Abelian $T$ dual of Klebanov-Tseytlin background
}

\author{
Sourav Roychowdhury $\oplus^{1, *}$ and Prasanta K. Tripathy $\oplus^{2, \dagger}$ \\ ${ }^{1}$ Chennai Mathematical Institute, SIPCOT IT Park, Siruseri 603 103, India \\ ${ }^{2}$ Department of Physics, Indian Institute of Technology Madras, Chennai 600 036, India
}

(Received 26 September 2021; accepted 10 November 2021; published 16 December 2021)

\begin{abstract}
In this paper we consider the Klebanov-Tseytlin background and its non-Abelian $T$-dual geometry along a suitably chosen $S U(2)$ subgroup of isometries. We analyze the Penrose limits along various null geodesics of both geometries. We observe that the Klebanov-Tseytlin geometry does not admit any pp-wave solutions. However, the $T$-dual background gives rise to a pp-wave solution upon taking the Penrose limit along some appropriate null geodesic. We comment on the possible gauge theory dual for our pp-wave background.
\end{abstract}

DOI: 10.1103/PhysRevD.104.126016

\section{INTRODUCTION}

String theory on a pp-wave background has been analyzed extensively during the past several decades because they are endowed with a number of unique features [1-5]. These pp-wave solutions appear as Penrose limits of various supergravity backgrounds in ten and eleven dimensions [6-8]. They provide exact string theory backgrounds to all orders in $\alpha^{\prime}$ as well as $g_{s}$ $[9,10]$. In the context of AdS/CFT correspondence these backgrounds give rise to the so-called BMN sector with large $R$-charge of the dual $\mathcal{N}=4$ superconformal theory in four dimensions [7]. The AdS/CFT correspondence is used to construct interacting string states from perturbative gauge theory [7].

Recently pp-wave backgrounds have been constructed from non-Abelian $T$-dual geometries of various supergravity theories. Non-Abelian $T$ duality has turned out to be a wonderful tool to construct new supergravity backgrounds from known ones. This is a nontrivial generalization of the conventional $T$ duality where a non-Abelian isometry group is used for dualization [11]. However, these non-Abelian $T$ dualities are not symmetries of the full string theory [12]. They are used to relate the low energy supergravity theories among each other. Originally non-Abelian $T$ duality was formulated for the NeveuSchwarz (NS) sector of supergravity theories. Subsequently, this formalism has been generalized to

\footnotetext{
souravroy@cmi.ac.in

prasanta@iitm.ac.in
}

Published by the American Physical Society under the terms of the Creative Commons Attribution 4.0 International license. Further distribution of this work must maintain attribution to the author(s) and the published article's title, journal citation, and DOI. Funded by SCOAP. include the Ramond-Ramond (RR) fields [13]. This in turn played a crucial role in relating different supergravity backgrounds among each other. Several examples of new supergravity backgrounds have also been constructed using the non-Abelian $T$ duality [14-21].

Of particular interest in the present context is the impact of these developments in understanding several aspects of AdS/CFT correspondence [22-29]. This has opened up the possibility of constructing several new CFT duals corresponding to these non-Abelian $T$-dual geometries. Relationships between a number of these dual geometries with the Penrose limits [30] of some of the prevailing supergravity backgrounds have also been revealed [21-23]. An important development in this context is the non-Abelian $T$ dual of type-IIB supergravity compactified on certain orbifolds of $\mathrm{AdS}_{5} \times S^{5}$ [27]. It has been shown that this geometry indeed admits plane wave solutions upon taking the Penrose limit along appropriate null geodesics [27]. A candidate for the field theory dual of this geometry has also been proposed. These developments have further been generalized for the non-Abelian $T$ duals of the KlebanovWitten background, which results in placing a stack of $D 3$ branes near a conifold singularity. The corresponding supergravity background, $\mathrm{AdS}_{5} \times T^{1,1}$, is obtained by blowing up the singularities of $\mathrm{AdS}_{5} \times S^{5}$ orbifolds [31,32]. An appropriate $S U(2)$ subrgoup of isometries of $T^{1,1}$ can be used to obtain the non-Abelian $T$-dual geometry [28]. These dual goemetries also give rise to pp-wave solutions upon considering the Penrose limits along appropriate null geodesics [33].

Placing a stack of $M$ fractional along with $N$ regular $D 3$ branes at the conifold singularity gives rise to an $\mathcal{N}=1$ supersymmetric $S U(N+M) \times S U(N)$ gauge theory. The gravity dual is a nontrivial modification of the $\mathrm{AdS}_{5} \times T^{1,1}$ background resulting in the well-known Klebanov-Tseytlin geometry [34]. The goemetry admits non-Abelian 
isometries, an $S U(2)$ subgroup of which is used to construct a non-Abelian $T$-dual background. This gives rise to a new massive type-IIA supergravity background $[16,24]$. In the present work we will analyze the Penrose limits of this massive type-IIA background in addition to the original Klebanov-Tseytlin background. We will show that, for the type-IIA theory, the resulting background indeed admits a pp-wave solution. The plan of this paper is as follows. In the next section we will review the KlebanovTseytlin background and its non-Abelian $T$ dual. Subsequently, in Sec. III we will analyze the Penrose limits and obtain the pp-wave solution. In Sec. IV we consider the supersymmetry analysis and show that the resulting pp-wave background preserves 16 supercharges. Finally, we will discuss some aspects of the dual quiver theory before summarizing the results.

\section{KLEBANOV-TSEYTLIN BACKGROUND}

Placing a stack of $N$ regular and $M$ fractional branes at a conifold singularity modifies the spacetime geometry in a nontrivial way. The gravity dual of this nonconformal $S U(N+M) \times S U(N)$ gauge theory has been accomplished in a pioneering work by Klebanov and Tseytlin [34]. The geometry of the resulting supergravity background is given by

$d s^{2}=H(r)^{-\frac{1}{2}} \eta_{\mu \nu} d x^{\mu} d x^{\nu}+H(r)^{\frac{1}{2}}\left(d r^{2}+r^{2} d s_{T^{1,1}}^{2}\right)$.

Here we will use the conventions of [35]. The warp factor is given by

$$
H(r)=\frac{1}{r^{4}}\left[R^{4}+2 L^{4}\left(\ln \left(\frac{r}{r_{0}}\right)+\frac{1}{4}\right)\right],
$$

with $\eta_{\mu \nu}$ denoting the stranded $(3+1)$-dimensional Minkowski metric. The metric of $T^{1,1}$ is given by [32]

$$
\begin{aligned}
d s_{T^{1,1}}^{2}= & \lambda_{1}^{2} d \Omega_{2}^{2}\left(\theta_{1}, \phi_{1}\right)+\lambda_{2}^{2} d \Omega_{2}^{2}\left(\theta_{2}, \phi_{2}\right) \\
& +\lambda^{2}\left(d \psi+\cos \theta_{1} d \phi_{1}+\cos \theta_{2} d \phi_{2}\right)^{2}
\end{aligned}
$$

In the above, $d \Omega_{2}^{2}(\theta, \phi)$ denotes the round metric on a twosphere. For $T^{1,1}$ the parameters $\lambda, \lambda_{1}, \lambda_{2}$ take the numerical values $\lambda_{1}^{2}=\lambda_{2}^{2}=\frac{1}{6}, \lambda^{2}=\frac{1}{9}$.
In addition, we need to specify nonvanishing background fields in NS-NS and RR sectors. The background NS-NS two-form field $B_{2}$ has the expression

$B_{2}=\frac{T(r)}{6 \sqrt{2}}\left(\sin \theta_{1} d \theta_{1} \wedge d \phi_{1}-\sin \theta_{2} d \theta_{2} \wedge d \phi_{2}\right)$,

with the corresponding field strengths

$H_{3}=\frac{L^{2}}{3 r}\left(\sin \theta_{1} d r \wedge d \theta_{1} \wedge d \phi_{1}-\sin \theta_{2} d r \wedge d \theta_{2} \wedge d \phi_{2}\right)$.

The nonvanishing RR fields strengths $F_{3}$ and $F_{5}$ are given respectively by

$$
\begin{aligned}
F_{3}= & \frac{P}{18 \sqrt{2}}\left(d \psi+\cos \theta_{1} d \phi_{1}+\cos \theta_{2} d \phi_{2}\right) \\
& \wedge\left(\sin \theta_{1} d \theta_{1} \wedge d \phi_{1}-\sin \theta_{2} d \theta_{2} \wedge d \phi_{2}\right),
\end{aligned}
$$

and

$$
F_{5}=\left(1+*_{10}\right) K(r) \operatorname{Vol}\left(T^{1,1}\right) .
$$

Here, $*_{10}$ denotes the Hodge dual with respect to the tendimensional metric (2.1). For convenience, in the above we have used the notation [34]

$$
\begin{aligned}
P & =\frac{L^{2}}{g_{s}} 2 \sqrt{2}, \\
T(r) & =2 \sqrt{2} L^{2} \ln \left(\frac{r}{r_{0}}\right), \\
K(r) & =\frac{r^{4}}{30} H(r)\left[1-\frac{L^{4}}{2 r^{4} H(r)}\right] .
\end{aligned}
$$

The numbers $N$ of regular $D 3$ branes, and $M$ of fractional $D 3$ branes corresponds respectively to the flux of $F_{5}$ and $F_{3}$. It is important to note that the constant $P$ is proportional to the number $M$ of fractional $D 3$ branes.

We will now consider the non-Abelian $T$ dual of the Klebanov-Tseytlin background. The non-Abelian $T$ duality with respect to an $S U(2)$ isometry has been obtained in [16,24]. The corresponding metric of the $T$-dual geometry is given by

$$
\begin{aligned}
d \hat{s}_{\mathrm{NATD}}^{2}= & H(r)^{-\frac{1}{2}} \eta_{\mu \nu} d x^{\mu} d x^{\nu}+H(r)^{\frac{1}{2}}\left(d r^{2}+\frac{1}{6} r^{2} d \Omega_{2}^{2}\left(\theta_{1}, \phi_{1}\right)\right)+\frac{1}{2 r^{2} \Delta H(r)^{\frac{1}{2}}}\left[12 r^{4} H(r) v_{2}^{2} \sigma_{\hat{3}}^{2}+12\left(r^{4} H(r)+27 v_{2}^{2}\right) d v_{2}^{2}\right. \\
& \left.+9\left(2 r^{4} H(r)+\mathcal{V}^{2}\right) d v_{3}^{2}+108 \mathcal{V} v_{2} d v_{2} d v_{3}\right],
\end{aligned}
$$


where the one form $\sigma_{\hat{3}}$ is defined as

$$
\sigma_{\hat{3}}=d \psi+\cos \theta_{1} d \phi_{1},
$$

and the functions $\Delta$ and $\mathcal{V}$ are given by

$$
\begin{aligned}
\hat{B}_{2}= & \frac{L^{2}}{3} \ln \left(\frac{r}{r_{0}}\right) \sin \theta_{1} d \theta_{1} \wedge d \phi_{1}+\frac{3 \sqrt{2}}{\Delta} \mathcal{V} v_{2} \sigma_{\hat{3}} \wedge d v_{2} \\
& +\frac{1}{\sqrt{2} \Delta}\left(2 r^{4} H(r)+\mathcal{V}^{2}\right) \sigma_{\hat{3}} \wedge d v_{3} \\
e^{-2 \hat{\Phi}}= & \frac{1}{81 g_{s}^{2}} r^{2} H(r)^{\frac{1}{2}} \Delta .
\end{aligned}
$$

The expression for the background NS-NS two form $\hat{B}_{2}$ and dilaton $\hat{\Phi}$ of the dual geometry are given by

$$
\begin{aligned}
\hat{F}_{0}= & -L^{2} \frac{2 \sqrt{2}}{9 g_{s}} \\
\hat{F}_{2}= & \frac{1}{162 \sqrt{2} g_{s}}\left[\frac{r^{4}}{5} H(r)\left(1-\frac{L^{4}}{2 r^{4} H(r)}\right)+L^{2} 6 \sqrt{2}\left(6 v_{3}+2 \sqrt{2} L^{2} \ln \left(\frac{r}{r_{0}}\right)\right)\right] \sin \theta_{1} d \theta_{1} \wedge d \phi_{1} \\
& -L^{2} \frac{4}{3 g_{s}} \frac{6 v_{3}+2 \sqrt{2} L^{2} \ln \left(\frac{r}{r_{0}}\right)}{\Delta} v_{2} \sigma_{\hat{3}} \wedge d v_{2}+L^{2} \frac{12}{g_{s}} \frac{v_{2}^{2}}{\Delta} \sigma_{\hat{3}} \wedge d v_{3}, \\
\hat{F}_{4}= & \frac{v_{2}}{18 \Delta g_{s}} \sin \theta_{1} d \theta_{1} \wedge d \phi_{1} \wedge d \psi \wedge\left[\left(-18 \sqrt{2} L^{2}\left(6 v_{3}+2 \sqrt{2} L^{2} \ln \left(\frac{r}{r_{0}}\right)\right)-\frac{3 r^{4}}{5} H(r)\left(1-\frac{L^{4}}{2 r^{4} H(r)}\right)\right) v_{2} d v_{3}\right. \\
& \left.+2\left(-2 \sqrt{2} L^{2} r^{4} H(r)+\frac{r^{4}}{30}\left(6 v_{3}+2 \sqrt{2} L^{2} \ln \left(\frac{r}{r_{0}}\right)\right) H(r)\left(1-\frac{L^{4}}{2 r^{4} H(r)}\right)-54 \sqrt{2} L^{2} v_{2}^{2}\right) d v_{2}\right] .
\end{aligned}
$$

\section{THE PENROSE LIMITS}

In this section we will study Penrose limits for both of the above backgrounds. We will first consider the original type-IIB background. The Penrose limit along a suitable null geodesics for this background has already been studied in [36]. Here we will first outline the main result of this work. Considering the motion of a massless particle in the $(r, \psi)$ plane of the background results in the following geometry:

$$
\begin{aligned}
d s^{2}= & 2 d u d v+\frac{r^{2}}{\sqrt{1+P \ln \left(\frac{r}{r_{0}}\right)}} d x_{3}^{2}+\sqrt{1+P \ln \left(\frac{r}{r_{0}}\right)}\left[1-\frac{\mu^{2} r^{2}}{1+P \ln \left(\frac{r}{r_{0}}\right)}\right] d x^{2} \\
& +\sqrt{1+P \ln \left(\frac{r}{r_{0}}\right)}\left[d r_{1}^{2}+r_{1}^{2} d \phi_{1}^{2}+d r_{2}^{2}+r_{2}^{2} d \phi_{2}^{2}\right]-\frac{\mu^{2}}{\sqrt{1+P \ln \left(\frac{r}{r_{0}}\right)}}\left(r_{1}^{2}+r_{2}^{2}\right) d u^{2} .
\end{aligned}
$$

The background gauge fields behave as

$$
\begin{aligned}
& B_{2} \sim P \ln \left(\frac{r}{r_{0}}\right)\left(d r_{1} \wedge r_{1} d \phi_{1}-d r_{2} \wedge r_{2} d \phi_{2}\right), \\
& F_{3} \sim P \dot{\psi} d u \wedge\left(d r_{1} \wedge r_{1} d \phi_{1}-d r_{2} \wedge r_{2} d \phi_{2}\right), \\
& F_{5} \sim(1+\star)\left(1+P \ln \left(\frac{r}{r_{0}}\right)\right) \dot{\psi} d u \wedge d r_{1} \wedge r_{1} d \phi_{1} \wedge d r_{2} \wedge r_{2} d \phi_{2} .
\end{aligned}
$$

It has been noted that [36] this background leads to ppwave upon setting $P=0$. As we noted earlier, the constant $P$ is proportional to $M$. Thus, setting $P$ to zero amounts to removing the fractional $D 3$ branes, thereby restoring to the undeformed Klebanov-Witten background. This indicates that the deformed background does not support a pp-wave upon taking Penrose limits. In Appendix A, we consider an extensive study of Penrose limits along the remaining null 
geodesics. Some of these geometries become singular whereas some others are smooth. Nevertheless none of these limits give rise to a pp-wave solution. However, as we will see in the following, this is not the case upon considering the non-Abelian $T$ duality. Thus, non-Abelian $T$ duality gives rise to new exactly solvable backgrounds that are absent in the original type IIB configuration.

In order to carry out the Penrose limit along appropriate null geodesics of the non-Abelian $T$-dual background, we will first rescale various quantities appropriately. Let us first consider the wrap factor $H(r)$. Introducing the parameter $\tilde{r}$ via

$$
\ln \tilde{r}=\ln r_{0}-\frac{1}{4}-\frac{R^{4}}{2 L^{4}},
$$

we can rewrite it as

$$
H(r)=\frac{2 L^{4}}{r^{4}} \ln \left(\frac{r}{\tilde{r}}\right)
$$

We will now rescale the Minkowski coordinates $x^{\mu} \rightarrow L^{2} x^{\mu}$ and the $T$-dual coordinates $v_{2,3} \rightarrow L^{2} v_{2,3}$. In terms of these rescaled coordinates, the $T$-dual metric (2.9) becomes

$$
\begin{aligned}
d \hat{s}_{\mathrm{NATD}}^{2}= & L^{2}\left[\frac{1}{\sqrt{2}} \frac{r^{2}}{\sqrt{\ln \left(\frac{r}{\tilde{r}}\right)}} \eta_{\mu \nu} d x^{\mu} d x^{\nu}+\sqrt{2} \frac{\sqrt{\ln \left(\frac{r}{\tilde{r}}\right)}}{r^{2}}\left(d r^{2}+\frac{1}{6} r^{2} d \Omega_{2}^{2}\left(\theta_{1}, \phi_{1}\right)\right)\right]+\frac{L^{2}}{A}\left[6 \sqrt{2} \sqrt{\ln \left(\frac{r}{\tilde{r}}\right)} v_{2}^{2} \sigma_{\hat{\mathrm{z}}}^{2}\right. \\
& +\left(6 \sqrt{2} \sqrt{\ln \left(\frac{r}{\tilde{r}}\right)}+\frac{81 \sqrt{2}}{\sqrt{\ln \left(\frac{r}{\tilde{r}}\right)}} v_{2}^{2}\right) d v_{2}^{2}+\left(9 \sqrt{2} \sqrt{\ln \left(\frac{r}{\tilde{r}}\right)}+\frac{9}{2 \sqrt{2}} \frac{1}{\sqrt{\ln \left(\frac{r}{\tilde{r}}\right)}}\left(6 v_{3}+2 \sqrt{2} \ln \left(\frac{r}{r_{0}}\right)\right)^{2}\right) d v_{3}^{2} \\
& \left.+\frac{27 \sqrt{2}}{\sqrt{\ln \left(\frac{r}{\tilde{r}}\right)}}\left(6 v_{3}+2 \sqrt{2} \ln \left(\frac{r}{r_{0}}\right)\right) v_{2} d v_{2} d v_{3}\right] .
\end{aligned}
$$

In the above, for easy reading we have introduced the notation

$$
A=4 \ln \left(\frac{r}{\tilde{r}}\right)+\left(6 v_{3}+2 \sqrt{2} \ln \left(\frac{r}{r_{0}}\right)\right)^{2}+54 v_{2}^{2} .
$$

After the rescaling, the NS-NS two-form $\hat{B}_{2}$ and the dilaton $\hat{\Phi}$ becomes

$$
\begin{aligned}
\hat{B}_{2}= & \frac{L^{2}}{3} \ln \left(\frac{r}{r_{0}}\right) \sin \theta_{1} d \theta_{1} \wedge d \phi_{1}+\frac{L^{2}}{A}\left[3 \sqrt{2}\left(6 v_{3}+2 \sqrt{2} \ln \left(\frac{r}{r_{0}}\right)\right) v_{2} \sigma_{\hat{3}} \wedge d v_{2}\right. \\
& \left.+\left(2 \sqrt{2} \ln \left(\frac{r}{\tilde{r}}\right)+\frac{1}{\sqrt{2}}\left(6 v_{3}+2 \sqrt{2} \ln \left(\frac{r}{r_{0}}\right)\right)^{2}\right) \sigma_{\hat{3}} \wedge d v_{3}\right], \\
e^{-2 \hat{\Phi}}= & \frac{L^{6}}{81 g_{s}^{2}} \sqrt{2} \sqrt{\ln \left(\frac{r}{\tilde{r}}\right)} A .
\end{aligned}
$$

Similarly, the field strengths in the RR sectors become

$$
\begin{aligned}
\hat{F}_{0}= & -L^{2} \frac{2 \sqrt{2}}{9 g_{s}} \\
\hat{F}_{2}= & \frac{L^{4}}{162 \sqrt{2} g_{s}}\left[\frac{2}{5} \ln \left(\frac{r}{\tilde{r}}\right)-\frac{1}{10}+6 \sqrt{2}\left(6 v_{3}+2 \sqrt{2} \ln \left(\frac{r}{r_{0}}\right)\right)\right] \sin \theta_{1} d \theta_{1} \wedge d \phi_{1} \\
& +\frac{L^{4}}{g_{s} A} \frac{4}{3}\left[-\left(6 v_{3}+2 \sqrt{2} \ln \left(\frac{r}{r_{0}}\right)\right) v_{2} \sigma_{\hat{3}} \wedge d v_{2}+9 v_{2}^{2} \sigma_{\hat{3}} \wedge d v_{3}\right], \\
\hat{F}_{4}= & \frac{L^{6}}{g_{s} A} v_{2} \sin \theta_{1} d \theta_{1} \wedge d \phi_{1} \wedge d \psi \wedge\left[\left(-\sqrt{2}\left(6 v_{3}+2 \sqrt{2} \ln \left(\frac{r}{r_{0}}\right)\right)-\frac{1}{15} \ln \left(\frac{r}{\tilde{r}}\right)+\frac{1}{60}\right) v_{2} d v_{3}\right. \\
& \left.+\left(-\frac{4 \sqrt{2}}{9} \ln \left(\frac{r}{\tilde{r}}\right)+\frac{1}{9}\left(\frac{1}{15} \ln \left(\frac{r}{\tilde{r}}\right)-\frac{1}{60}\right)\left(6 v_{3}+2 \sqrt{2} \ln \left(\frac{r}{r_{0}}\right)\right)-6 \sqrt{2} v_{2}^{2}\right) d v_{2}\right] .
\end{aligned}
$$


We will now consider the Penrose limits of the above $T$-dual background along appropriate null geodesics. Denoting the spacetime coordinates as $\left\{x^{\mu}\right\}$, the geodesic equation is expressed as

$$
\frac{d^{2} x^{\mu}}{d u^{2}}+\Gamma_{\nu \rho}^{\mu} \frac{d x^{\nu}}{d u} \frac{d x^{\rho}}{d u}=0
$$

Here we use $u$ to denote the affine parameter along the geodesic. We are interested to examine the motion along various isometry directions. Denote $x^{\lambda}$ one such isometry direction. Thus, we need to set the velocity and acceleration along any direction $x^{\mu}, \mu \neq \lambda$ zero:

$$
\frac{d x^{\mu}}{d u}=0=\frac{d^{2} x^{\mu}}{d u^{2}}, \quad \text { for } \mu \neq \lambda .
$$

Substituting the above in (3.7), we find that the geodesic equation for motion along an isometry direction takes the simple form

$$
\partial^{\mu} g_{\lambda \lambda}=0
$$

In addition to the above condition, we need to impose $d s^{2}=0$ in order to obtain null geodesics for our purpose.

Let us now focus on various isometry directions of the $T$-dual geometry. A quick inspection of the rescaled geometry (3.4) indicates that both $\psi$ and $\phi_{1}$ are isometry directions. Let us first consider the motion along the $\psi$ direction. The geodesics equation for this case is

$$
\partial_{\mu} g_{\psi \psi}=0 .
$$

From (3.4) we note the relevant component of the metric:

$$
g_{\psi \psi \psi}=\frac{L^{2}}{A} 6 \sqrt{2} \sqrt{\ln \left(\frac{r}{\tilde{r}}\right)} v_{2}^{2} .
$$

The metric component $g_{\psi \psi}$ depends upon $r, v_{2}$, and $v_{3}$. For $\mu=r$, the geodesic condition (3.10) leads to $v_{2}=0$. Similarly, for $\mu=v_{2}, v_{3}$ we obtain $\left\{r=\tilde{r}, v_{2}=0\right\}$. However, for all the above values, the metric component $g_{\psi \mu \psi}$ in (3.11) vanishes leading to singular geometries. In the following, we will no longer consider Penrose limits for such singular geometries.

We will now consider motion along the $\phi_{1}$ direction. Consider the metric component $g_{\phi_{1} \phi_{1}}$ along the $\phi_{1}$ direction:

$g_{\phi_{1} \phi_{1}}=L^{2} \sqrt{\ln \left(\frac{r}{\tilde{r}}\right)}\left[\frac{1}{3 \sqrt{2}} \sin ^{2} \theta_{1}+\frac{6 \sqrt{2}}{A} v_{2}^{2} \cos ^{2} \theta_{1}\right]$.

Let us analyze in detail the geodesic condition:

$$
\partial_{\mu} g_{\phi_{1} \phi_{1}}=0
$$

For $\mu=r$, the above equation leads to $\left\{\theta_{1}=(0, \pi)\right.$, $\left.v_{2}=0\right\}$. For $\mu=\theta_{1}$, we find $\left\{r=\tilde{r}, \theta_{1}=\left(0, \frac{\pi}{2}, \pi\right)\right\}$. On the other hand, for $\mu=v_{2}, v_{3}$ we obtain $\{r=\tilde{r}$, $\left.\theta_{1}=\frac{\pi}{2}, v_{2}=0\right\}$. The geodesic condition is trivially satisfied for all other values of $\mu$. Consider the values $r=\tilde{r}$, $\left\{\theta_{1}=(0, \pi), v_{2}=0\right\}$. The metric component $g_{\phi_{1} \phi_{1}}$ in (3.12) vanishes for all these cases. Thus, they lead to singular geometries. We will not consider these geodesics any more.

Finally, we will consider the Penrose limit around $\theta_{1}=\frac{\pi}{2}, v_{2}=0=v_{3}$, keeping the $r$ coordinate constant, i.e., $r=c$ for some constant $c \neq \tilde{r} \neq 0$. Consider the following expansion around this geodesic:

$$
\begin{aligned}
x_{i} & =\frac{y_{i}}{L} ; \quad i=1,2,3, \quad r=c+\frac{x}{L}, \\
\theta_{1} & =\frac{\pi}{2}+\frac{z}{L}, \quad t=a x^{+}, \quad \phi_{1}=b x^{+}+\frac{x^{-}}{L^{2}} .
\end{aligned}
$$

In addition, we do the rescaling of the coordinates $v_{2}$ and $v_{3}$ as $v_{2} \rightarrow \frac{v_{2}}{L}, \quad v_{3} \rightarrow \frac{v_{3}}{L}$ while keeping the $\psi$ coordinate unchanged. In the above, $a$ and $b$ are some constant parameters. The null geodesic condition relates the parameters $a, b$, and $c$ as

$$
a^{2}=\frac{b^{2}}{3 c^{2}} \ln \left(\frac{c}{\tilde{r}}\right)
$$

Using the above expansion we consider the leading terms of the $T$-dual metric in the limit $L \rightarrow \infty$. We find

$$
\begin{aligned}
d s^{2}= & \frac{1}{3 \sqrt{2}} \sqrt{\ln \left(\frac{c}{\tilde{r}}\right)} 2 b d x^{+} d x^{-}+\frac{1}{\sqrt{2}} \frac{c^{2}}{\sqrt{\ln \left(\frac{c}{\tilde{r}}\right)}}\left(d y_{1}^{2}+d y_{2}^{2}+d y_{3}^{2}\right)+\frac{\sqrt{2}}{c^{2}} \sqrt{\ln \left(\frac{c}{\tilde{r}}\right)} d x^{2} \\
& +\frac{1}{3 \sqrt{2}} \sqrt{\ln \left(\frac{c}{\tilde{r}}\right)} d z^{2}+\frac{3}{\sqrt{2}} \frac{\sqrt{\ln \left(\frac{c}{\tilde{r}}\right)}}{\ln \left(\frac{c}{\tilde{r}}\right)+2\left(\ln \left(\frac{c}{r_{0}}\right)\right)^{2}}\left(d v_{2}^{2}+v_{2}^{2} d \psi^{2}\right)+\frac{9}{2 \sqrt{2}} \frac{1}{\sqrt{\ln \left(\frac{c}{\tilde{r}}\right)}} d v_{3}^{2} \\
& -\frac{b^{2}}{3 \sqrt{2} c^{2}} \sqrt{\ln \left(\frac{c}{\tilde{r}}\right)}\left[\frac{x^{2}}{\ln \left(\frac{c}{\tilde{r}}\right)}+x^{2}+c^{2} z^{2}\right]\left(d x^{+}\right)^{2}-L \frac{2 b^{2} x}{3 c} \ln \left(\frac{c}{\tilde{r}}\right)\left(d x^{+}\right)^{2} .
\end{aligned}
$$


This contains a divergent term which cannot be removed for any choice of the parameter $b$. Note that, from the null geodesic condition (3.14), setting $b=0$ is not allowed. Hence, motion along the isometric direction $\phi_{1}$ by keeping $r=$ constant does not lead to any smooth geometry.

We can repeat similar analysis for motion along the isometry direction $\psi$. Recall that the $\psi \psi$ component of the $T$-dual metric is given as

$$
g_{\psi \psi \psi}=\frac{L^{2}}{A} 6 \sqrt{2} \sqrt{\ln \left(\frac{r}{\tilde{r}}\right)} v_{2}^{2} .
$$

The null geodesic condition

$$
\partial_{\mu} g_{\psi \psi}=0
$$

for $\mu=r$ leads to $v_{2}=0$, whereas for $\mu=v_{2}, v_{3}$ we obtain $\left\{r=\tilde{r}, v_{2}=0\right\}$. However, the metric component $g_{\psi \mu}$ vanishes for all these values. Thus, we do not have a regular geometry for any of the above geodesics.

To the end we will consider null geodesics for the motion of a particle carrying nonzero angular momentum in the $\left(r, \phi_{1}\right)$ plane. We will subject our analysis to a small neighborhood of $\theta_{1}=\frac{\pi}{2}$ and $v_{2}=v_{3}=0$. Consider the Lagrangian for a massless particle moving along this geodesic:

$$
\mathcal{L}=\frac{1}{2} g_{\mu \nu} \dot{X}^{\mu} \dot{X}^{\nu}
$$

Let $u$ be the affine parameter along the geodesic. The dots in the above equation correspond to the derivative with respect to $u$. Substituting the explicit expression for the background metric (3.4) in the above Lagrangian we find

$$
\begin{aligned}
\mathcal{L}= & \frac{L^{2}}{2}\left(-\frac{1}{\sqrt{2}} \frac{r^{2}}{\sqrt{\ln \left(\frac{r}{\tilde{r}}\right)}} \dot{t}^{2}+\frac{\sqrt{2}}{r^{2}} \sqrt{\ln \left(\frac{r}{\tilde{r}}\right)} \dot{r}^{2}\right. \\
& \left.+\frac{1}{3 \sqrt{2}} \sqrt{\ln \left(\frac{r}{\tilde{r}}\right)} \dot{\phi}_{1}^{2}\right) .
\end{aligned}
$$

We will now obtain the conserved quantities corresponding to the above system. Note that the Lagrangian (3.19) does not depend on the generalized coordinates $t$ and $\phi_{1}$ explicitly. Denoting $-E L^{2}$ to be the conserved momentum associated with $t$, we find

$$
E=-\frac{1}{L^{2}} \frac{\partial \mathcal{L}}{\partial \dot{t}}=\frac{r^{2}}{\sqrt{2 \ln \left(\frac{r}{\tilde{r}}\right)}} \dot{t} .
$$

Similarly, let $-J L^{2}$ be the conserved momentum associated with the generalized coordinate $\phi_{1}$. We find

$$
J=-\frac{1}{L^{2}} \frac{\partial \mathcal{L}}{\partial \dot{\phi}_{1}}=-\frac{1}{3 \sqrt{2}} \sqrt{\ln \left(\frac{r}{\tilde{r}}\right)} \dot{\phi}_{1}
$$

In addition, we will require the geodesic to be null. This gives rise to the condition:

$$
\dot{r}^{2}+\frac{3 r^{2}}{\ln \left(\frac{r}{\tilde{r}}\right)} J^{2}=E^{2}
$$

We will now concentrate on obtaining the Penrose limit for a null geodesic carrying angular momentum $J$ around $x_{i}=0, i=1,2,3, \theta_{1}=\frac{\pi}{2}$ and $v_{2}=v_{3}=0$. We redefine the coordinates as

$$
\begin{aligned}
x_{i} & =\frac{y_{i}}{L} ; & i=1,2,3, & \theta_{1}=\frac{\pi}{2}+\frac{z}{L}, \\
v_{2} & \rightarrow \frac{v_{2}}{L}, & v_{3} & \rightarrow \frac{v_{3}}{L} .
\end{aligned}
$$

We keep the $\psi$ coordinate unchanged, and redefine the string coupling as $g_{s}=L^{3} \tilde{g}_{s}$, in order to keep the dilaton finite at the Penrose limit. Finally, we will consider the following expansion in the limit $L \rightarrow \infty$ :

$$
\begin{aligned}
d t & =c_{1} d u, \quad d r=c_{2} d u+c_{3} \frac{d w}{L}, \\
d \phi_{1} & =c_{4} d u+c_{5} \frac{d w}{L}+c_{6} \frac{d v}{L^{2}} .
\end{aligned}
$$

We need to determine the coefficients $c_{i}$. Requiring the geodesic to be null determines the values of the coefficients $c_{1}, c_{2}$, and $c_{4}$ as follows:

$$
\begin{aligned}
& c_{1}=\frac{E \sqrt{2}}{r^{2}} \sqrt{\ln \left(\frac{r}{\tilde{r}}\right)}, \\
& c_{2}=\left[E^{2}-\frac{3 r^{2}}{\ln \left(\frac{r}{\tilde{r}}\right)} J^{2}\right]^{\frac{1}{2}}, \\
& c_{4}=-\frac{3 \sqrt{2}}{\sqrt{\ln \left(\frac{r}{\tilde{r}}\right)}} J .
\end{aligned}
$$

We now substitute the expansion (3.24) in the $T$-dual metric (3.4) and retain the leading terms. A priori, this metric will contain divergent terms of order $L$ and $L^{2}$. Imposing the null geodesic condition automatically cancels the $\mathcal{O}\left(L^{2}\right)$ terms. It can be easily verified that the $\mathcal{O}(L)$ term is removed upon setting

$$
c_{2} c_{3}+\frac{r^{2}}{6} c_{4} c_{5}=0
$$

Using the value of $c_{2}$ and $c_{4}$ from (3.25) in the above equation we can express the coefficient $c_{3}$ in terms $c_{5}$ as 


$$
c_{3}=\left[E^{2}-\frac{3 r^{2}}{\ln \left(\frac{r}{\tilde{r}}\right)} J^{2}\right]^{-\frac{1}{2}} \frac{r^{2}}{\sqrt{2 \ln \left(\frac{r}{\tilde{r}}\right)}} J c_{5}
$$

We will see later that the coefficient $c_{5}$ can be determined by requiring the background fields to satisfy Einstein's equations. Finally, we need to determine the coefficient $c_{6}$. This is easily obtained by setting appropriate normalization for the cross term $d u d v$ in the metric. We find

$$
c_{6}=-\frac{1}{J}
$$

Substituting the above results in (3.4), and taking the limit $L \rightarrow \infty$, we find the pp-wave metric of the form

$$
\begin{aligned}
d s^{2}= & 2 d u d v+\frac{1}{\sqrt{2}} \frac{r^{2}}{\sqrt{\ln \left(\frac{r}{\tilde{r}}\right)}}\left(d y_{1}^{2}+d y_{2}^{2}+d y_{3}^{2}\right)+\sqrt{\ln \left(\frac{r}{\tilde{r}}\right)}\left(\frac{c_{3}^{2} \sqrt{2}}{r^{2}}+\frac{c_{5}^{2}}{3 \sqrt{2}}\right) d w^{2}+\frac{1}{3 \sqrt{2}} \sqrt{\ln \left(\frac{r}{\tilde{r}}\right)} d z^{2} \\
& +\frac{3}{\sqrt{2}} \frac{\sqrt{\ln \left(\frac{r}{\tilde{r}}\right)}}{\ln \left(\frac{r}{\tilde{r}}\right)+2\left(\ln \left(\frac{r}{r_{0}}\right)\right)^{2}}\left(d v_{2}^{2}+v_{2}^{2} d \psi^{2}\right)+\frac{9}{2 \sqrt{2}} \frac{1}{\sqrt{\ln \left(\frac{r}{\tilde{r}}\right)}} d v_{3}^{2}-\frac{3 \sqrt{2}}{\sqrt{\ln \left(\frac{r}{\tilde{r}}\right)}} J^{2} z^{2} d u^{2} .
\end{aligned}
$$

We will subsequently show that this is indeed a pp-wave solution by rewriting it in the standard Brinkmann form. Let us now consider the Penrose limit for the remaining background fields. In this limit, the NS-NS two-form field and dilaton takes the form

$$
\begin{aligned}
\hat{B}_{2} & =\frac{c_{5}}{3} \ln \left(\frac{r}{r_{0}}\right) d z \wedge d w-3 \frac{\ln \left(\frac{r}{r_{0}}\right)}{\ln \left(\frac{r}{\tilde{r}}\right)+2\left(\ln \left(\frac{r}{r_{0}}\right)\right)^{2}} v_{2} d v_{2} \wedge d \psi+\frac{3 J z}{\sqrt{\ln \left(\frac{r}{\tilde{r}}\right)}} d u \wedge d v_{3}, \\
e^{-2 \hat{\Phi}} & =\frac{4 \sqrt{2}}{81 \tilde{g}_{s}^{2}} \sqrt{\ln \left(\frac{r}{\tilde{r}}\right)}\left[\ln \left(\frac{r}{\tilde{r}}\right)+2\left(\ln \left(\frac{r}{r_{0}}\right)\right)^{2}\right] .
\end{aligned}
$$

The field strengths corresponding to the RR sector are given by

$$
\begin{aligned}
& \hat{F}_{0}=0, \\
& \hat{F}_{2}=\frac{J}{54 \tilde{g}_{s}} \frac{1}{\sqrt{\ln \left(\frac{r}{\tilde{r}}\right)}}\left[\frac{2}{5} \ln \left(\frac{r}{\tilde{r}}\right)+24 \ln \left(\frac{r}{r_{0}}\right)-\frac{1}{10}\right] d u \wedge d z, \\
& \hat{F}_{4}=\frac{2 J}{3 \tilde{g}_{s}\left(\ln \left(\frac{r}{\tilde{r}}\right)+2\left(\ln \left(\frac{r}{r_{0}}\right)\right)^{2}\right)} \frac{1}{\sqrt{\ln \left(\frac{r}{\tilde{r}}\right)}} v_{2}\left[-\ln \left(\frac{r}{\tilde{r}}\right)+\frac{1}{2} \ln \left(\frac{r}{r_{0}}\right)\left(\frac{1}{15} \ln \left(\frac{r}{\tilde{r}}\right)-\frac{1}{60}\right)\right] d u \wedge d z \wedge d \psi \wedge d v_{2} .
\end{aligned}
$$

For later use, we will also compute the field strength $\hat{H}_{3}$ corresponding to the NS-NS two-form $\hat{B}_{2}$ :

$$
\begin{aligned}
\hat{H}_{3}= & \frac{1}{3}\left[E^{2}-\frac{3 r^{2}}{\ln \left(\frac{r}{\tilde{r}}\right)} J^{2}\right]^{\frac{1}{2}}\left[c_{5}^{\prime} \ln \left(\frac{r}{r_{0}}\right)+\frac{c_{5}}{r}\right] d u \wedge d z \wedge d w-3 v_{2}\left[E^{2}-\frac{3 r^{2}}{\ln \left(\frac{r}{\tilde{r}}\right)} J^{2}\right]^{\frac{1}{2}} \\
& \frac{\ln \left(\frac{r}{\tilde{r}}\right)-\ln \left(\frac{r}{r_{0}}\right)-2\left(\ln \left(\frac{r}{r_{0}}\right)\right)^{2}}{r\left(\ln \left(\frac{r}{\tilde{r}}\right)+2\left(\ln \left(\frac{r}{r_{0}}\right)\right)^{2}\right)^{2}} d u \wedge d v_{2} \wedge d \psi+\frac{3 J}{\sqrt{\ln \left(\frac{r}{\tilde{r}}\right)}} d u \wedge d v_{3} \wedge d z .
\end{aligned}
$$

Note that, in obtaining the above, we have used $d r=c_{2} d u$ where the expression for the coefficient $c_{2}$ is given by (3.25).

As pointed out earlier, the metric obtained in (3.29) is not in the standard Brinkmann form [5]. A formalism has been developed in [27] in order to transform the line element to the Brinkmann form. Following [27] consider a line element of the form

$$
d s^{2}=2 d u d v+\sum_{i} A_{i}(u) d x_{i}^{2} .
$$

Now, replace the coordinates $x_{i}$ and $v$ as

$$
x_{i} \rightarrow \frac{x_{i}}{\sqrt{A_{i}}}, \quad v \rightarrow v+\frac{1}{4} \sum_{i} \frac{\dot{A_{i}}}{A_{i}} x_{i}^{2} .
$$


The line element in (3.33) now takes the Brinkmann form

$$
d s^{2}=2 d u d v+\sum_{i} d x_{i}^{2}+\left(\sum_{i} F_{i}(u) x_{i}^{2}\right) d u^{2}
$$

$$
F_{i}=\frac{1}{4} \frac{\dot{A_{i}^{2}}}{A_{i}^{2}}+\frac{1}{2} \frac{d}{d u}\left(\frac{\dot{A_{i}}}{A_{i}}\right)
$$

with the functions $F_{i}$ being

For the case of our pp-wave metric (3.29) we have

$$
\begin{aligned}
& A_{y_{1}}=A_{y_{2}}=A_{y_{3}}=\frac{1}{\sqrt{2}} \frac{r^{2}}{\sqrt{\ln \left(\frac{r}{\tilde{r}}\right)}}, \quad A_{w}=\sqrt{\ln \left(\frac{r}{\tilde{r}}\right)}\left(\frac{c_{3}^{2} \sqrt{2}}{r^{2}}+\frac{c_{5}^{2}}{3 \sqrt{2}}\right), \\
& A_{z}=\frac{1}{3 \sqrt{2}} \sqrt{\ln \left(\frac{r}{\tilde{r}}\right)}, \quad A_{v_{2}}=\frac{3}{\sqrt{2}} \frac{\sqrt{\ln \left(\frac{r}{\tilde{r}}\right)}}{\ln \left(\frac{r}{\tilde{r}}\right)+2\left(\ln \left(\frac{r}{r_{0}}\right)\right)^{2}}, \quad A_{v_{3}}=\frac{9}{2 \sqrt{2}} \frac{1}{\sqrt{\ln \left(\frac{r}{\tilde{r}}\right)}} .
\end{aligned}
$$

Hence after making the following replacement

$$
\begin{aligned}
y_{1} & \rightarrow \frac{y_{1}}{\sqrt{A_{y_{1}}}}, \quad y_{2} \rightarrow \frac{y_{2}}{\sqrt{A_{y_{2}}}}, \quad y_{3} \rightarrow \frac{y_{3}}{\sqrt{A_{y_{3}}},} \quad w \rightarrow \frac{w}{\sqrt{A_{w}}}, \quad z \rightarrow \frac{z}{\sqrt{A_{z}}}, \\
v_{2} & \rightarrow \frac{v_{2}}{\sqrt{A_{v_{2}}}}, \quad v_{3} \rightarrow \frac{v_{3}}{\sqrt{A_{v_{3}}}} \quad \text { and } \\
v & \rightarrow v+\frac{1}{4}\left[\frac{\dot{A}_{y_{1}}}{A_{y_{1}}} y_{1}^{2}+\frac{\dot{A}_{y_{2}}}{A_{y_{2}}} y_{2}^{2}+\frac{\dot{A}_{y_{3}}}{A_{y_{3}}} y_{3}^{2}+\frac{\dot{A}_{w}}{A_{w}} w^{2}+\frac{\dot{A}_{z}}{A_{z}} z^{2}+\frac{\dot{A}_{v_{2}}}{A_{v_{2}}} v_{2}^{2}+\frac{\dot{A}_{v_{3}}}{A_{v_{3}}} v_{3}^{2}\right],
\end{aligned}
$$

we find

$$
\begin{aligned}
d s^{2}= & 2 d u d v+d y_{1}^{2}+d y_{2}^{2}+d y_{3}^{2}+d w^{2}+d z^{2}+d v_{2}^{2}+v_{2}^{2} d \psi^{2}+d v_{3}^{2} \\
& +\left[F_{y_{1}} y_{1}^{2}+F_{y_{2}} y_{2}^{2}+F_{y_{3}} y_{3}^{2}+F_{w} w^{2}+F_{z} z^{2}+F_{v_{2}} v_{2}^{2}+F_{v_{3}} v_{3}^{2}-\frac{3 \sqrt{2}}{\sqrt{\ln \left(\frac{r}{\tilde{r}}\right)}} J^{2} z^{2}\right] d u^{2},
\end{aligned}
$$

where the functions $F_{i}$ can be read from the expression (3.36).

We will now express the background fields in the Brinkmann form. The dilaton $\hat{\Phi}$ and the NS-NS three-form flux $\hat{H}_{3}$ are given as

$$
\begin{aligned}
e^{-2 \hat{\Phi}}= & \frac{4 \sqrt{2}}{81 \tilde{g}_{s}^{2}} \sqrt{\ln \left(\frac{r}{\tilde{r}}\right)}\left[\ln \left(\frac{r}{\tilde{r}}\right)+2\left(\ln \left(\frac{r}{r_{0}}\right)\right)^{2}\right], \\
\hat{H}_{3}= & \frac{2^{\frac{1}{4}}}{\sqrt{3}}\left[E^{2}-\frac{3 r^{2}}{\ln \left(\frac{r}{\tilde{r}}\right)} J^{2}\right]^{\frac{1}{2}}\left[c_{5}^{\prime} \ln \left(\frac{r}{r_{0}}\right)+\frac{c_{5}}{r}\right]\left[\frac{c_{3}^{2} \sqrt{2}}{r^{2}}+\frac{c_{5}^{2}}{3 \sqrt{2}}\right]^{-\frac{1}{2}} \frac{1}{\sqrt{\ln \left(\frac{r}{\tilde{r}}\right)}} d u \wedge d z \wedge d w \\
& -\sqrt{2} v_{2}\left[E^{2}-\frac{3 r^{2}}{\ln \left(\frac{r}{\tilde{r}}\right)} J^{2}\right]^{\frac{1}{2} \ln \left(\frac{r}{\tilde{r}}\right)-\ln \left(\frac{r}{r_{0}}\right)-2\left(\ln \left(\frac{r}{r_{0}}\right)\right)^{2}} \frac{1}{r\left(\ln \left(\frac{r}{\tilde{r}}\right)+2\left(\ln \left(\frac{r}{r_{0}}\right)\right)^{2}\right)} \frac{1}{\sqrt{\ln \left(\frac{r}{\tilde{r}}\right)}} d u \wedge d v_{2} \wedge d \psi+\frac{2 \sqrt{3} J}{\sqrt{\ln \left(\frac{r}{\tilde{r}}\right)}} d u \wedge d v_{3} \wedge d z .
\end{aligned}
$$

Similarly, the expressions for the RR field strengths are found to be of the form

$$
\begin{aligned}
& \hat{F}_{0}=0 \\
& \hat{F}_{2}=\frac{2^{\left(-\frac{3}{4}\right)}}{9 \sqrt{3} \tilde{g}_{s}} \frac{J}{\left(\ln \left(\frac{r}{\tilde{r}}\right)^{\frac{3}{4}}\right.}\left[\frac{2}{5} \ln \left(\frac{r}{\tilde{r}}\right)+24 \ln \left(\frac{r}{r_{0}}\right)-\frac{1}{10}\right] d u \wedge d z \\
& \hat{F}_{4}=\frac{2^{\frac{7}{4}}}{3 \sqrt{3} \tilde{g}_{s}} \frac{J}{\left(\ln \left(\frac{r}{\tilde{r}}\right)^{\frac{5}{4}}\right.} v_{2}\left[-\ln \left(\frac{r}{\tilde{r}}\right)+\frac{1}{2} \ln \left(\frac{r}{r_{0}}\right)\left(\frac{1}{15} \ln \left(\frac{r}{\tilde{r}}\right)-\frac{1}{60}\right)\right] d u \wedge d z \wedge d \psi \wedge d v_{2} .
\end{aligned}
$$


We will now verify that these fields indeed satisfy the Bianchi identities and the gauge field equation of motion. A quick inspection of the background fields in (3.40)-(3.41) shows that the Bianchi identities

$d \hat{H}_{3}=0, \quad d \hat{F}_{2}=\hat{F}_{0} \hat{H}_{3}, \quad d \hat{F}_{4}=\hat{H}_{3} \wedge \hat{F}_{2}$

hold. The field strengths $\hat{H}_{3}, \hat{F}_{2}$, and $\hat{F}_{4}$ are all closed and both $\hat{F}_{0}$ as well as $\hat{H}_{3} \wedge \hat{F}_{2}$ are indeed zero.
Let us now inspect the type-IIA supergravity equations for the gauge fields

$$
\begin{aligned}
d\left(e^{-2 \hat{\Phi}} \hat{H}_{3}\right)-\hat{F}_{2} \wedge \star \hat{F}_{4}-\frac{1}{2} \hat{F}_{4} \wedge \hat{F}_{4}=\hat{F}_{0} \star \hat{F}_{2}, \\
d \star \hat{F}_{2}+\hat{H}_{3} \wedge \star \hat{F}_{4}=0, \\
d \star \hat{F}_{4}+\hat{H}_{3} \wedge \hat{F}_{4}=0 .
\end{aligned}
$$

The Hodge duals for the above background fields are

$$
\begin{aligned}
\star \hat{H}_{3}= & \frac{1}{7 !} d u \wedge d y_{1} \wedge d y_{2} \wedge d y_{3}\left[\frac{2^{\frac{1}{4}}}{\sqrt{3}} v_{2}\left(E^{2}-\frac{3 r^{2}}{\ln \left(\frac{r}{\tilde{r}}\right)} J^{2}\right)^{\frac{1}{2}}\left(c_{5}^{\prime} \ln \left(\frac{r}{r_{0}}\right)+\frac{c_{5}}{r}\right)\left(\frac{c_{3}^{2} \sqrt{2}}{r^{2}}+\frac{c_{5}^{2}}{3 \sqrt{2}}\right)^{-\frac{1}{2}} \frac{1}{\sqrt{\ln \left(\frac{r}{\tilde{r}}\right)}} d v_{2} \wedge d \psi \wedge d v_{3}\right. \\
& \left.-\sqrt{2}\left(E^{2}-\frac{3 r^{2}}{\ln \left(\frac{r}{\tilde{r}}\right)} J^{2}\right)^{\frac{1}{2} \ln \left(\frac{r}{\tilde{r}}\right)-\ln \left(\frac{r}{r_{0}}\right)-2\left(\ln \left(\frac{r}{r_{0}}\right)\right)^{2}} \frac{1}{r\left(\ln \left(\frac{r}{\tilde{r}}\right)+2\left(\ln \left(\frac{r}{r_{0}}\right)\right)^{2}\right)^{2}} \frac{1}{\sqrt{\ln \left(\frac{r}{\tilde{r}}\right)}} d z \wedge d w \wedge d v_{3}+v_{2} \frac{2 \sqrt{3} J}{\sqrt{\ln \left(\frac{r}{\tilde{r}}\right)}} d w \wedge d v_{2} \wedge d \psi\right], \\
\star \hat{F}_{2}= & \frac{1}{8 !} \frac{2^{\left(-\frac{3}{4}\right)}}{9 \sqrt{3} \tilde{g}_{s}} \frac{J}{\left(\ln \left(\frac{r}{\tilde{r}}\right)\right)^{\frac{3}{4}}} v_{2}\left[\frac{2}{5} \ln \left(\frac{r}{\tilde{r}}\right)+24 \ln \left(\frac{r}{r_{0}}\right)-\frac{1}{10}\right] d u \wedge d y_{1} \wedge d y_{2} \wedge d y_{3} \wedge d w \wedge d \psi \wedge d v_{2} \wedge d v_{3}, \\
\star \hat{F}_{4}= & \frac{1}{6 !} \frac{2^{\frac{7}{4}}}{3 \sqrt{3} \tilde{g}_{s}} \frac{J}{\left(\ln \left(\frac{r}{\tilde{r}}\right)\right)^{\frac{5}{4}}}\left[-\ln \left(\frac{r}{\tilde{r}}\right)+\frac{1}{2} \ln \left(\frac{r}{r_{0}}\right)\left(\frac{1}{15} \ln \left(\frac{r}{\tilde{r}}\right)-\frac{1}{60}\right)\right] d u \wedge d y_{1} \wedge d y_{2} \wedge d y_{3} \wedge d w \wedge d v_{3} .
\end{aligned}
$$

In deriving the above, we have used

$$
\begin{aligned}
\operatorname{det}\left(g_{p p}\right) & =-v_{2}^{2}, \quad g^{v v}=-g_{u u}, \\
g^{u v} & =g^{v u}=1, \quad g^{i j}=\delta^{i j}\left(\text { only } g^{\psi \psi}=\frac{1}{v_{2}^{2}}\right) .
\end{aligned}
$$

It is straightforward to see that the background fields (3.40)-(3.41) together with (3.44) indeed satisfy the gauge field equations for type-IIA supergravity. Both $\star \hat{H}_{3}$ as well as $e^{-2 \hat{\Phi}} \star \hat{H}_{3}$ are closed. Also, $\hat{F}_{2} \wedge \star \hat{F}_{4}$ and $\hat{F}_{4} \wedge \hat{F}_{4}$ vanish identically. Thus, the first of the equations in (3.43) is satisfied. Similarly, both $\star \hat{F}_{2}$ and $\star \hat{F}_{4}$ are exact forms. In addition, $\hat{H}_{3} \wedge \hat{F}_{4}$ and $\hat{H}_{3} \wedge \star \hat{F}_{4}$ vanish as well. Thus, the last two equations in (3.43) are also satisfied.

It is interesting to note that the gauge field equations as well as the Bianchi identities hold irrespective of the value of the coefficient $c_{5}$. However, as we will see in the following, this is not the case with Einstein's equations. For type-IIA supergravity, Einstein's equations are given as

$$
\begin{aligned}
\hat{R}_{\mu \nu}+2 D_{\mu} D_{\nu} \hat{\Phi}= & \frac{1}{4} \hat{H}_{\mu \nu}^{2}+e^{2 \hat{\Phi}}\left[\frac{1}{2}\left(\hat{F}_{2}^{2}\right)_{\mu \nu}+\frac{1}{12}\left(\hat{F}_{4}^{2}\right)_{\mu \nu}\right. \\
& \left.-\frac{1}{4} g_{\mu \nu}\left(\hat{F}_{0}^{2}+\frac{1}{2} \hat{F}_{2}^{2}+\frac{1}{4 !} \hat{F}_{4}^{2}\right)\right] .
\end{aligned}
$$

Similarly, the dilation equations are

$$
\hat{R}+4 D^{2} \hat{\Phi}-4(\partial \hat{\Phi})^{2}-\frac{1}{12} \hat{H}^{2}=0 .
$$

In the Appendix B we have analyzed these equations in detail. We find that the equation of motion for dilation (3.46) holds automatically. In addition, we observe that Einstein's equations (3.45) are trivially satisfied for all values of $\mu, \nu$ except for $\mu=\nu=u$. In this case we have the nontrivial condition

$\hat{R}_{u u}+2 D_{u} D_{u} \hat{\Phi}=\frac{1}{4} \hat{H}_{u u}^{2}+e^{2 \hat{\Phi}}\left[\frac{1}{2}\left(\hat{F}_{2}^{2}\right)_{u u}+\frac{1}{12}\left(\hat{F}_{4}^{2}\right)_{u u}\right]$.

This equation involves the undetermined coefficient $c_{5}$. We can solve this equation to determine the expression for the coefficient $c_{5}$. The analysis in Appendix B outlines the steps to determine it.

\section{SUPERSYMMETRY OF pp-WAVE}

The supersymmatry analysis of non-Abelian $T$-dual backgrounds has been studied extensively [14-16,37-39]. Unlike the $\mathrm{AdS}_{5} \times S^{5}$ case, the non-Abelian $T$ dual of Klebanov-Witten as well as the Klebanov-Tseytlin background preserves all the supersymmetries of the original background. This is because in the later two cases, the Killing spinor of the original background does not carry 
any $S U(2)$ charge of the isometry group used for nonAbelian $T$ dualization [14,39]. In this context, it is worth investigating whether the pp-wave we obtained in the above preserves any supersymmetry.

In order to analyze this, we will first introduce the Brinkmann coordinates $X_{i}$ such that

$$
\begin{aligned}
d y_{i}^{2} & =\left(d X^{i}\right)^{2} ; \quad i=1,2,3, \quad w=X^{4}, \quad z=X^{5}, \\
d v_{2}^{2}+v_{2}^{2} d \psi^{2} & =\left(d X^{6}\right)^{2}+\left(d X^{7}\right)^{2}, \quad v_{3}=X^{8} .
\end{aligned}
$$

In these coordinates the pp-wave background (3.39)-(3.41) reads as

$$
\begin{aligned}
d s^{2} & =2 d u d v+\sum_{i=1}^{8} d X_{i}^{2}+\mathcal{H} d u^{2}, \\
\hat{\Phi} & =\Phi(u), \\
\hat{H}_{3} & =f_{1}(u) d u \wedge d X^{5} \wedge d X^{4}-f_{2}(u) d u \wedge d X^{6} \wedge d X^{7}+f_{3}(u) d u \wedge d X^{8} \wedge d X^{5}, \\
\hat{F}_{2} & =f_{4}(u) d u \wedge d X^{5}, \\
\hat{F}_{4} & =f_{5}(u) d u \wedge d X^{5} \wedge d X^{7} \wedge d X^{6},
\end{aligned}
$$

where we have introduced the notation

$$
\begin{aligned}
\mathcal{H}= & F_{i j} X^{i} X^{j}=\left[F_{X^{1}}\left(X^{1}\right)^{2}+F_{X^{2}}\left(X^{2}\right)^{2}+F_{X^{3}}\left(X^{3}\right)^{2}+F_{X^{4}}\left(X^{4}\right)^{2}+\left(F_{X^{5}}-\frac{3 \sqrt{2}}{\sqrt{\ln \left(\frac{r}{\tilde{r}}\right)}} J^{2}\right)\left(X^{5}\right)^{2}\right. \\
& \left.+F_{X^{6}}\left(X^{6}\right)^{2}+F_{X^{7}}\left(X^{7}\right)^{2}+F_{X^{8}}\left(X^{8}\right)^{2}\right], \\
f_{1}(u)= & \frac{2^{\frac{1}{4}}}{\sqrt{3}}\left[E^{2}-\frac{3 r^{2}}{\ln \left(\frac{r}{\tilde{r}}\right)} J^{2}\right]^{\frac{1}{2}}\left[c_{5}^{\prime} \ln \left(\frac{r}{r_{0}}\right)+\frac{c_{5}}{r}\right]\left[\frac{c_{3}^{2} \sqrt{2}}{r^{2}}+\frac{c_{5}^{2}}{3 \sqrt{2}}\right]^{-\frac{1}{2}} \frac{1}{\sqrt{\ln \left(\frac{r}{\tilde{r}}\right)}}, \\
f_{2}(u)= & \sqrt{2}\left[E^{2}-\frac{3 r^{2}}{\ln \left(\frac{r}{\tilde{r}}\right)} J^{2}\right]^{\frac{1}{2} \ln \left(\frac{r}{\tilde{r}}\right)-\ln \left(\frac{r}{r_{0}}\right)-2\left(\ln \left(\frac{r}{r_{0}}\right)\right)^{2}} \frac{1}{r\left(\ln \left(\frac{r}{\tilde{r}}\right)+2\left(\ln \left(\frac{r}{r_{0}}\right)\right)^{2}\right)} \frac{1}{\sqrt{\ln \left(\frac{r}{\tilde{r}}\right)}}, \\
f_{3}(u)= & \frac{2 \sqrt{3} J}{\sqrt{\ln \left(\frac{r}{\tilde{r}}\right)}}, \\
f_{4}(u)= & \frac{2^{\left(-\frac{3}{4}\right)}}{9 \sqrt{3} \tilde{g}_{s}} \frac{J}{\left(\ln \left(\frac{r}{\tilde{r}}\right)\right)^{\frac{3}{4}}}\left[\frac{2}{5} \ln \left(\frac{r}{\tilde{r}}\right)+24 \ln \left(\frac{r}{r_{0}}\right)-\frac{1}{10}\right], \\
f_{5}(u)= & \frac{2^{\frac{7}{4}}}{3 \sqrt{3} \tilde{g}_{s}} \frac{J}{\left(\ln \left(\frac{r}{\tilde{r}}\right)\right)^{\frac{5}{4}}}\left[-\ln \left(\frac{r}{\tilde{r}}\right)+\frac{1}{2} \ln \left(\frac{r}{r_{0}}\right)\left(\frac{1}{15} \ln \left(\frac{r}{\tilde{r}}\right)-\frac{1}{60}\right)\right] .
\end{aligned}
$$

The functions $F_{i j}$ are defined by

$$
\begin{aligned}
& F_{11}=F_{22}=F_{33}=F_{X^{i}} ; \quad i=1,2,3, \\
& F_{44}=F_{X^{4}}, \quad F_{55}=F_{X^{5}}-\frac{3 \sqrt{2}}{\sqrt{\ln \left(\frac{r}{\tilde{r}}\right)}} J^{2}, \\
& F_{66}=F_{X^{6}}, \quad F_{77}=F_{X^{7}}, \quad F_{88}=F_{X^{8}} .
\end{aligned}
$$

Now we introduce the frame $\left\{e^{a}\right\}$ as $e^{-}=d u, \quad e^{+}=d v+\frac{1}{2} \mathcal{H} d u, \quad e^{i}=d X^{i}$, such that the pp-wave metric (4.2) can be written as

$$
d s^{2}=2 e^{+} e^{-}+\sum_{i=1}^{8}\left(e^{i}\right)^{2}=\eta_{a b} e^{a} e^{b},
$$

with $\eta_{+-}=\eta_{-+}=1$ and $\eta_{i j}=\delta_{i j}$. The nonvanishing components of spin connections are given by

$$
\omega_{-i}=-\omega_{i-}=\omega^{+i}=-\omega^{i+}=\frac{1}{2} \partial_{i} \mathcal{H} d u .
$$

In terms of the frame (4.5), the background fields (4.2) take form 


$$
\begin{aligned}
\hat{\Phi} & =\Phi(u), \\
\hat{H}_{3} & =f_{1}(u) e^{-} \wedge e^{5} \wedge e^{4}-f_{2}(u) e^{-} \wedge e^{6} \wedge e^{7}+f_{3}(u) e^{-} \wedge e^{8} \wedge e^{5}, \\
\hat{F}_{2} & =f_{4}(u) e^{-} \wedge e^{5}, \\
\hat{F}_{4} & =f_{5}(u) e^{-} \wedge e^{5} \wedge e^{7} \wedge e^{6} .
\end{aligned}
$$

We will now analyze the spinor conditions in detail. Consider the supersymmetric variations of the dilatino and gravitino

$$
\begin{aligned}
\delta \hat{\lambda} & =\frac{1}{2} \not \partial \hat{\Phi} \hat{\epsilon}-\frac{1}{24} \hat{H} \sigma_{3} \hat{\epsilon}+\frac{1}{8} e^{\hat{\Phi}}\left[\frac{3}{2} \hat{F}_{2}\left(i \sigma_{2}\right)+\frac{1}{24} \hat{F}_{4} \sigma_{1}\right] \hat{\epsilon}, \\
\delta \hat{\psi}_{\mu} & =D_{\mu} \hat{\epsilon}-\frac{1}{8} \hat{H}_{\mu \nu \rho} \Gamma^{\nu \rho} \sigma_{3} \hat{\epsilon}+\frac{1}{8} e^{\hat{\Phi}}\left[\frac{1}{2} \hat{F}_{2}\left(i \sigma_{2}\right)+\frac{1}{24} \hat{F}_{4} \sigma_{1}\right] \Gamma_{\mu} \hat{\epsilon} .
\end{aligned}
$$

Here we follow the conventions of [15,27]. In particular, we have the covariant derivative $D_{\mu} \hat{\epsilon}=\partial_{\mu} \hat{\epsilon}+\frac{1}{4} \omega_{\mu}^{a b} \Gamma_{a b} \hat{\epsilon}$, and in addition we use the notation $\hat{F}_{n} \equiv \hat{F}_{i_{1} \ldots i_{n}} \Gamma^{i_{1} \ldots i_{n}}$. In the above, $\sigma_{i}$ denote the Pauli matrices. The Killing spinor $\hat{\epsilon}$ consists of real Majorana-Weyl spinors $\hat{\epsilon}_{ \pm}$, such that

$$
\hat{\epsilon}=\left(\begin{array}{c}
\hat{\epsilon}_{+} \\
\hat{\epsilon}_{-}
\end{array}\right) .
$$

In type-IIA supergravity $\hat{\epsilon}$ satisfies $\Gamma_{11} \hat{\epsilon}=-\sigma_{3} \hat{\epsilon}$. We also introduce

$$
\Gamma^{ \pm}=\frac{1}{\sqrt{2}}\left(\Gamma^{9} \pm \Gamma^{0}\right)
$$

$\mathcal{R}=\frac{1}{4}\left(f_{1}(u)\left(\delta_{i 4} \Gamma^{5}-\delta_{i 5} \Gamma^{4}\right)-f_{2}(u)\left(\delta_{i 7} \Gamma^{6}-\delta_{i 6} \Gamma^{7}\right)+f_{3}(u)\left(\delta_{i 5} \Gamma^{8}-\delta_{i 8} \Gamma^{5}\right)\right) \sigma_{3}-\frac{e^{\hat{\Phi}}}{8}\left(f_{4}(u) \Gamma^{5}\left(i \sigma_{2}\right)+f_{5}(u) \Gamma^{576} \sigma_{1}\right) \Gamma^{i}$.

Now, $\Gamma^{-}$anticommutes with $\mathcal{R}$ and $\Gamma^{-} \hat{\epsilon}=0$. Thus, we have $\partial_{i} \hat{\epsilon}=0$ leading to $\hat{\epsilon}=\chi(u)$ for some $\chi(u)$ satisfying $\Gamma^{-} \chi(u)=0$. Finally, we consider the variation $\delta \hat{\psi}_{-}=0$. Note that in this case the covariant derivative $D_{-}$becomes

$$
D_{-}=\partial_{-}+\frac{1}{2} F_{i j} X^{j} \Gamma^{-i}
$$

After some simplification, we find that the condition $\delta \hat{\psi}_{-}=0$ gives rise to

$$
\begin{gathered}
\partial_{u} \chi(u)-\frac{1}{4}\left(f_{1}(u) \Gamma^{54}-f_{2}(u) \Gamma^{67}+f_{3}(u) \Gamma^{85}\right) \sigma_{3} \chi(u) \\
-\frac{e^{\hat{\Phi}}}{4}\left(f_{4}(u) \Gamma^{5}\left(i \sigma_{2}\right)+f_{5}(u) \Gamma^{576} \sigma_{1}\right) \chi(u)=0
\end{gathered}
$$

We now proceed to solve the spinor conditions. Substituting the background fields in (4.9), and setting the dilatino variation to zero, we obtain after some simplification

$$
\begin{gathered}
\Gamma^{-}\left[\dot{\hat{\Phi}}-\frac{1}{2}\left(f_{1}(u) \Gamma^{54}-f_{2}(u) \Gamma^{67}+f_{3}(u) \Gamma^{85}\right) \sigma_{3}\right. \\
\left.+\frac{e^{\hat{\Phi}}}{4}\left(3 f_{4}(u) \Gamma^{5}\left(i \sigma_{2}\right)+f_{5}(u) \Gamma^{576} \sigma_{1}\right)\right] \hat{\epsilon}=0 .
\end{gathered}
$$

The above condition holds provided $\Gamma^{-} \hat{\epsilon}=0$. This indicates that, subject to the compatibility with the gravitino variation, the pp-wave background (4.2) preserves 16 supercharges. We now proceed to verify the spinor condition arising from the variation of the gravitino. Let us first consider the $\delta \hat{\psi}_{+}$variation. The NS-NS three-form does not have any leg along $e^{+}$. Together with $\Gamma_{+} \hat{\epsilon}=\Gamma^{-} \hat{\epsilon}=0$, the variation $\delta \hat{\psi}_{+}=0$ leads to $\partial_{+} \hat{e}=0$. Thus, we find that the Killing spinor $\hat{\epsilon}$ is independent of $v$, i.e., $\hat{\epsilon}=\hat{\epsilon}\left(u, X^{i}\right)$.

Now we focus on the variation $\delta \hat{\psi}_{i}, i=1, \ldots, 8$. The vanishing of $\delta \hat{\psi}_{i}$ implies that

$$
\partial_{i} \hat{\epsilon}=\Gamma^{-} \mathcal{R} \hat{\epsilon},
$$

where we have introduced the notation
This is of the form $\partial_{u} \chi(u)-\mathcal{M}(u) \chi(u)=0$, which can be integrated to give rise

$$
\chi(u)=e^{\int d u \mathcal{M}(u)} \chi_{0} .
$$

This proves that the gravitino condition is compatible with the dilatino variation for the above choice of $\chi(u)$, provided $\Gamma^{-} \chi_{0}=0$. Thus, from the above analysis we find that the pp-wave background (4.9) indeed preserves 16 supercharges.

\section{GAUGE THEORY DUALS}

It is well known that the field theory dual to the Klebanov-Tseytlin geometry consists of a nonconformal $\mathcal{N}=1 \quad$ supersymmetric $S U(N+M) \times S U(N)$ gauge 
theory [34]. It describes the dynamics of $N$ regular and $M$ fractional $D 3$ branes placed near a conifold singularity. The fractional $D 3$ branes arise due to $D 5$ branes wrapping the vanishing two-cycle at conifold singularity. The nonconformal gauge theory has a nontrivial renormalization group (RG) flow. Near UV the supergravity description is valid and the dual geometry is given by the Klebanov-Tseytlin background. As the theory is flown to IR it undergoes to a cascade of Seiberg dualities thereby changing the number of $D 3$ branes from $N$ to $N-M$ in each step, resulting in a singular geometry at the end. However, for a suitably chosen initial condition the conifold geometry gets deformed at IR by strong coupling effects thereby leading to the Klebanov-Strassler background [40].

For the non-Abelian $T$-dual geometry of the KlebanovTseytlin background the field theory dual has been considered in [16,24]. The existence of domain wall configurations plays a key role in understanding the dual field theories. In the type-IIB theory domain walls can be formed by wrapping $D 5$ branes on suitably chosen two cycles of the internal manifold. For the Klebanov-Tseytlin background (2.1)-(2.4) one such two-cycle can be constructed upon the identification

$$
\theta_{1}=\theta_{2}, \quad \phi_{1}=2 \pi-\phi_{2}, \quad \psi=\psi_{0},
$$

with a constant $\psi_{0}$. This gives rise to the following twocycle $\Sigma_{2}$ for the $T$-dual background (3.4)-(3.6):

$$
\Sigma_{2}=\left[\theta_{1}, \phi_{1}\right], \quad v_{2}=v_{3}=\psi=0 .
$$

Furthermore, it is possible to construct a three-cycle $\Sigma_{3}$ in the $T$-dual geometry as

$$
\Sigma_{3}=\left[\theta_{1}, \phi_{1}, \psi\right], \quad v_{2}=v_{3}=r=\text { constant }
$$

Following [24] we will now analyze the construction of domain wall in the Klebanov-Tseytlin background and its $T$ dual. For the Klebanov-Tseytlin background we will consider the domain wall formed by a $D 5$ brane extended along $\mathbb{R}^{1,2} \in \mathbb{R}^{1,3}$ of the (1,3) Minkowski spacetime and wrapping the compact directions parametrized by $\left\{\theta_{2}, \phi_{2}, \psi\right\}$. The dynamics of the low energy excitations are captured in terms of the corresponding Born-Infeld action on the world volume of the $D 5$ brane. This gives rise to the corresponding effective tension.

The non-Abelian $T$ duality is performed along an $S U(2)$ isometry parametrized by the coordinates $\left\{\theta_{2}, \phi_{2}, \psi\right\}$. Thus the $D 5$ brane wrapping the $S U(2)$ directions gives rise to a $D 2$ brane extending along the $\mathbb{R}^{1,2}$ of the $T$-dual geometry. We will place this domain wall at the origin of the internal manifold:

$$
v_{2}=v_{3}=\theta_{1}=\phi_{1}=\psi=0 .
$$

Once again we can consider the corresponding Born-Infeld action and compute the effective tension for it. The effective tension of the domain wall in the KlebanovTesytlin background matches with the effective tension of the corresponding configuration in the $T$-dual geometry up to a constant factor [24]. In addition, it has been shown that [24] the central charge as well as entanglement entropy of both the theories match up to an renormaliztion group independent coefficient. While $T$ duality maintains the essential features of the central charge and entanglement entropy, this is not the case for the four-dimensional gauge coupling. The $T$-dual geometry gives rise to a very unusual behavior for the gauge coupling. It has been demonstrated that $1 / g^{2} \sim(\ln r)^{3 / 2}$, unlike the case for a conventional field theory where a logarithmic behavior is observed.

The Maxwell and Page charges of the $D$-branes in the theory also play a significant role in understanding the field theory dual. For the Klebanov-Tseytlin background we consider D3 and D5 brane charges

$$
\begin{aligned}
& Q_{\mathrm{Max}, \mathrm{D} 3}=\frac{1}{2 k_{10}^{2} T_{D 3}} \int_{T^{1,1}} F_{5}=\frac{K(r)}{27 \pi}, \\
& Q_{\mathrm{Max}, \mathrm{D} 5}=\frac{1}{2 k_{10}^{2} T_{D 5}} \int_{\theta_{2}, \phi_{2}, \psi} F_{3}=\frac{\sqrt{2} P}{9},
\end{aligned}
$$

and

$$
\begin{gathered}
Q_{\mathrm{Page}, \mathrm{D} 3}=\frac{1}{2 k_{10}^{2} T_{D 3}} \int_{T^{1,1}} F_{5}-B_{2} \wedge F_{3}=\frac{Q(r)}{27 \pi}, \\
Q_{\mathrm{Page}, \mathrm{D} 5}=\frac{1}{2 k_{10}^{2} T_{D 5}} \int_{\theta_{2}, \phi_{2}, \psi} F_{3}-B_{2} \wedge F_{1}=\frac{\sqrt{2} P}{9},
\end{gathered}
$$

where $Q(r)=K(r)+P T(r)$ and we choose the normalization factor as described in [18].

For the $T$-dual background, the Maxwell and Page charges of $D 6$ and $D 8$ are given respectively as

$$
\begin{aligned}
& \hat{Q}_{\mathrm{Max}, \mathrm{D} 6}=\frac{1}{\sqrt{2} \pi^{2}} \int_{\theta_{1}, \phi_{1}} \hat{F}_{2}=\frac{K(r)+Q(r)}{27 \pi}, \\
& \hat{Q}_{\mathrm{Max}, \mathrm{D} 8}=\sqrt{2} \int \hat{F}_{0}=\frac{\sqrt{2} P}{9},
\end{aligned}
$$

and

$$
\begin{aligned}
& \hat{Q}_{\text {Page, D6 }}=\frac{1}{\sqrt{2} \pi^{2}} \int_{\theta_{1}, \phi_{1}} \hat{F}_{2}-\hat{B}_{2} \hat{F}_{0}=\frac{2 Q(r)}{27 \pi}, \\
& \hat{Q}_{\text {Page, D8 }}=\sqrt{2} \int \hat{F}_{0}=\frac{\sqrt{2} P}{9} .
\end{aligned}
$$

The above shows that, after dualization we find $D 8$ branes for each of the $D 5$ branes and twice the number of $D 6$ branes for each of the $D 3$ branes in the original 
background. It has been noticed in [24] that the changes induced in the page charge of the $D 3$ brane in the Klebanov-Tseytlin background by a large gauge transformation of the NS-NS two from $B_{2}$ is the same as the changes in the Maxwell charge by a suitable change in the radial coordinate. A similar phenomenon is observed in the dual gauge theory, where the page charge of the $D 6$ brane now undergoes a shift under the large gauge transformation. This suggests that the quiver theory corresponding to the $T$-dual geometry undergoes to a cascade of
Seiberg dualities much the same way as the gauge theory corresponding to the original geometry. Since the change in D6 brane charge is twice the change in the D3 charge, the $T$-dual theory undergoes a Seiberg duality by a change of $2 M$ units of $D 6$ brane charge for a change of $M$ units of the $D 3$ brane charge in the Klebanov-Tseytlin background.

We will now consider the Maxwell and Page charges for pp-wave background. Recall that the RR field strengths for this background in Brinkmann coordinates are given as

$$
\begin{aligned}
& \hat{F}_{0}=0 \\
& \hat{F}_{2}=\frac{2^{\left(-\frac{3}{4}\right)}}{9 \sqrt{3} \tilde{g}_{s}} \frac{J}{\left(\ln \left(\frac{r}{\tilde{r}}\right)\right)^{\frac{3}{4}}}\left[\frac{2}{5} \ln \left(\frac{r}{\tilde{r}}\right)+24 \ln \left(\frac{r}{r_{0}}\right)-\frac{1}{10}\right] d u \wedge d z, \\
& \hat{F}_{4}=\frac{2^{\frac{7}{4}}}{3 \sqrt{3} \tilde{g}_{s}} \frac{J}{\left(\ln \left(\frac{r}{\tilde{r}}\right)\right)^{\frac{5}{4}}} v_{2}\left[-\ln \left(\frac{r}{\tilde{r}}\right)+\frac{1}{2} \ln \left(\frac{r}{r_{0}}\right)\left(\frac{1}{15} \ln \left(\frac{r}{\tilde{r}}\right)-\frac{1}{60}\right)\right] d u \wedge d z \wedge d \psi \wedge d v_{2} .
\end{aligned}
$$

The Maxwell and page charge for various branes in type-IIA theory is given by

$$
\begin{aligned}
& \hat{Q}_{\text {Max }, \mathrm{D} 6}=\frac{1}{\sqrt{2} \pi^{2}} \int \hat{F}_{2}, \\
& \hat{Q}_{\text {Max }, \mathrm{D} 8}=\sqrt{2} \int \hat{F}_{0},
\end{aligned}
$$

and

$$
\begin{aligned}
& \hat{Q}_{\text {Page }, \mathrm{D} 6}=\frac{1}{\sqrt{2} \pi^{2}} \int \hat{F}_{2}-\hat{B}_{2} \hat{F}_{0}, \\
& \hat{Q}_{\text {Page }, \mathrm{D} 8}=\sqrt{2} \int \hat{F}_{0} .
\end{aligned}
$$

Since $\hat{F}_{0}$ is zero for our background, the $D 8$ charges are all zero. Moreover, the Maxwell and Page charges for $D 6$ branes are both equal. The Maxwell and Page charges for $D 2$ branes also vanish. We have

$$
\begin{aligned}
\hat{Q}_{\mathrm{Max}, \mathrm{D} 2}= & \frac{1}{2 k_{10}^{2} T_{D 2}} \int_{c_{6}} \hat{F}_{6}, \\
\hat{Q}_{\text {Page, D2 }}= & \frac{1}{2 k_{10}^{2} T_{D 2}} \int_{c_{6}}\left[\hat{F}_{6}-\hat{B}_{2} \wedge \hat{F}_{4}+\frac{1}{2} \hat{B}_{2} \wedge \hat{B}_{2} \wedge \hat{F}_{2}\right. \\
& \left.-\frac{1}{6} \hat{F}_{0} \hat{B}_{2} \wedge \hat{B}_{2} \wedge \hat{B}_{2}\right] .
\end{aligned}
$$

For fixed $v_{2}$, both $\hat{F}_{4}$ and $\hat{F}_{6}$ are zero and $\hat{F}_{0}$ as well as $\hat{B}_{2} \wedge \hat{F}_{2}$ vanish for the pp-wave background. From (5.9), we find that there is no longer any cascading due to large gauge transformation of $\hat{B}_{2}$. This indicates that the quiver theory dual to the pp-wave geometry corresponds to the end point of the cascade.

\section{CONCLUSION}

In this paper we have considered Penrose limits for the Klebaov-Tseytlin geometry and its non-Abelian $T$ dual around a suitable $S U(2)$ isometry. We have scrutinized various null geodesics in these geometries. A direct investigation of the Penrose limits for the KlebanovTseytlin geometry gives rise to singular geometries for most of the null geodesics. We found one smooth geometry with a nonvanishing scalar curvature. However, taking the non-Abelian $T$ duality results in a pp-wave solution around a suitably chosen null geodesic. The holographic dual of the $T$-dual background exhibits a cascade of Seiberg dualities under large gauge transformation of the NS-NS two form thereby reducing the number of $D 6$ branes in each step. However, an analysis of the Maxwell and Page charges shows the absence of similar phenomenon for the pp-wave background. Thus, the holographic dual in this case appears to be the end point of the cascade of quivers corresponding to the $T$-dual geometry. The gauge coupling analysis shows that the quiver in the $T$-dual case is a nonconventional field theory. Further investigation is required to precisely identify the quiver and also the corresponding BMN sector and to establish a map between holographic quantities and field theory observables. It would also be interesting to explore the possibility of obtaining pp-wave geometries for the non-Abelian $T$ dual of Klebanov-Strassler background as well as backgrounds with $\mathrm{AdS}_{3}$ factors. Dualization of the Baryonic branch of the Klebanov-Strassler geometry has already been carried out [29]. We hope to address some of these issues in the future. 


\section{APPENDIX A: PENROSE LIMITS IN KLEBANOV-TSEYTLIN BACKGROUND}

In this Appendix we will examine Penrose limits for various null geodesics in the Klebanov-Tseytlin geometry. Recall the metric of Klebanov-Tseytlin background

$d s^{2}=H(r)^{-\frac{1}{2}} \eta_{\mu \nu} d x^{\mu} d x^{\nu}+H(r)^{\frac{1}{2}}\left(d r^{2}+r^{2} d s_{T^{1,1}}^{2}\right)$,

with the warp factor

$H(r)=\frac{1}{r^{4}}\left[R^{4}+2 L^{4}\left(\ln \left(\frac{r}{r_{0}}\right)+\frac{1}{4}\right)\right]=\frac{2 L^{4}}{r^{4}} \ln \left(\frac{r}{\tilde{r}}\right)$.

The $T^{1,1}$ metric is given by

$$
\begin{aligned}
d s_{T^{1,1}}^{2}= & \lambda_{1}^{2} d \Omega_{2}^{2}\left(\theta_{1}, \phi_{1}\right)+\lambda_{2}^{2} d \Omega_{2}^{2}\left(\theta_{2}, \phi_{2}\right) \\
& +\lambda^{2}\left(d \psi+\cos \theta_{1} d \phi_{1}+\cos \theta_{2} d \phi_{2}\right)^{2} .
\end{aligned}
$$

The parameters $\lambda, \lambda_{1}, \lambda_{2}$ in the $T^{1,1}$ metric have the numerical values $\lambda_{1}^{2}=\lambda_{2}^{2}=\frac{1}{6}$ and $\lambda^{2}=\frac{1}{9}$.

We will now rescale the Minkowski coordinates $\left(x^{\mu}\right)$ as $x^{\mu} \rightarrow L^{2} x^{\mu}$. The metric then becomes

$$
\begin{aligned}
d s^{2}= & L^{2}\left[\frac{1}{\sqrt{2}} \frac{r^{2}}{\sqrt{\ln \left(\frac{r}{\tilde{r}}\right)}} \eta_{\mu \nu} d x^{\mu} d x^{\nu}+\frac{\sqrt{2}}{r^{2}} \sqrt{\ln \left(\frac{r}{\tilde{r}}\right)} d r^{2}\right. \\
& \left.+\sqrt{2} \sqrt{\ln \left(\frac{r}{\tilde{r}}\right)} d s_{T^{1,1}}^{2}\right] .
\end{aligned}
$$

The above metric has $U(1)$ isometries along $\phi_{1}, \phi_{2}$, and $\psi$ directions. We will examine the Penrose limits by considering the motion along these isometry directions. Let us first consider motion along the $\psi$ direction. The $\psi \psi$ component of the metric is given by

$$
g_{\psi \mu}=\lambda^{2} L^{2} \sqrt{2} \sqrt{\ln \left(\frac{r}{\tilde{r}}\right)} .
$$

Now by imposing the null geodesic condition we get

$$
\lambda^{2} L^{2} \frac{1}{\sqrt{2} r \sqrt{\ln \left(\frac{r}{\tilde{r}}\right)}}=0
$$

which does not admit any smooth solution.

Now we shall consider motion along the $\phi_{1}$ direction. The relevant metric component is

$$
g_{\phi_{1} \phi_{1}}=L^{2} \sqrt{2} \sqrt{\ln \left(\frac{r}{\tilde{r}}\right)}\left[\lambda_{1}^{2} \sin ^{2} \theta_{1}+\lambda^{2} \cos ^{2} \theta_{1}\right] .
$$

The geodesic condition for $\mu=r$ does not give any solution. For $\mu=\theta_{1}$ the condition leads to the solution $r=\tilde{r}$ and $\theta_{1}=\left\{0, \frac{\pi}{2}, \pi\right\}$. However, $r=\tilde{r}$ is a singular point as the metric component $g_{\phi_{1} \phi_{1}}$ vanishes for this value of $r$. Hence we shall consider the Penrose limit around $\theta_{1}=$ $\left\{0, \frac{\pi}{2}, \pi\right\}, \theta_{2}=\phi_{2}=\psi=0$ while the $r$ coordinate fixed.

Consider the following expansion around the geodesic $\theta_{1}=\theta_{2}=\phi_{2}=\psi=0$ and $r=$ constant:

$$
\begin{aligned}
x_{i} & =\frac{y_{i}}{L} ; \quad i=1,2,3, \quad r=c+\frac{w}{L}, \quad \theta_{1}=\frac{z}{L}, \quad \theta_{2}=\frac{x}{L}, \\
t & =a x^{+}, \quad \phi_{1}=b x^{+}+\frac{x^{-}}{L^{2}}, \quad \phi_{2} \rightarrow \frac{\phi_{2}}{L}, \quad \psi \rightarrow \frac{\psi}{L},
\end{aligned}
$$

where $a, b$, and $c$ are some nonvanishing parameters. The null geodesic condition gives

$$
a^{2}=\frac{2 \lambda^{2} b^{2}}{c^{2}} \ln \left(\frac{c}{\tilde{r}}\right)
$$

For the above expansion, the leading terms of the $T$-dual metric in the limit $L \rightarrow \infty$ are given by

$$
\begin{aligned}
d s^{2}= & 2 \sqrt{2} \lambda^{2} b \sqrt{\ln \left(\frac{c}{\tilde{r}}\right)} d x^{+} d x^{-}+\frac{1}{\sqrt{2}} \frac{c^{2}}{\sqrt{\ln \left(\frac{c}{\tilde{r}}\right)}}\left(d y_{1}^{2}+d y_{2}^{2}+d y_{3}^{2}\right)+\frac{\sqrt{2}}{c^{2}} \sqrt{\ln \left(\frac{c}{\tilde{r}}\right)} d w^{2} \\
& +\lambda_{1}^{2} \sqrt{2} \sqrt{\ln \left(\frac{c}{\tilde{r}}\right)} d z^{2}+\lambda_{2}^{2} \sqrt{2} \sqrt{\ln \left(\frac{c}{\tilde{r}}\right)} d x^{2}+\lambda^{2} \sqrt{2} \sqrt{\ln \left(\frac{c}{\tilde{r}}\right)}\left(\left(d \psi+d \phi_{2}\right)^{2}\right. \\
& \left.+\frac{w b}{c \ln \left(\frac{c}{\tilde{r}}\right)} d x^{+} d \psi+2 b d x^{+} d \phi_{2}\right)+\left[\frac{1}{\sqrt{2}} \frac{-w^{2} a^{2}}{\sqrt{\ln \left(\frac{c}{\tilde{r}}\right)}}\left(\frac{1}{4 \ln \left(\frac{c}{\tilde{r}}\right)}+1\right)+\sqrt{2} \sqrt{\ln \left(\frac{c}{\tilde{r}}\right)} b^{2} z^{2}\left(\lambda_{1}^{2}-\lambda^{2}\right)\right. \\
& \left.-\frac{w^{2} b^{2}}{4 c^{2} \ln \left(\frac{c}{\tilde{r}}\right)}\right]\left(d x^{+}\right)^{2}+L\left[\frac{1}{\sqrt{2}} \frac{c w a^{2}}{\sqrt{\ln \left(\frac{c}{\tilde{r}}\right)}}\left(\frac{1}{2 \ln \left(\frac{c}{\tilde{r}}\right)}-2\right)\left(d x^{+}\right)^{2}+2 \sqrt{2} \lambda^{2} b \sqrt{\ln \left(\frac{c}{\tilde{r}}\right)} d x^{+} d \psi\right] .
\end{aligned}
$$


We can see that the divergent term of order $\mathcal{O}(L)$ cannot be removed for any choice of the parameters $a, b, c$.

Now we consider the following expansion around the geodesic $\theta_{1}=\frac{\pi}{2}$ and $\theta_{2}=\phi_{2}=\psi=0$ and $r=$ constant:

$$
\begin{aligned}
x_{i} & =\frac{y_{i}}{L} ; \quad i=1,2,3, \quad r=c+\frac{w}{L}, \quad \theta_{1}=\frac{\pi}{2}+\frac{z}{L}, \quad \theta_{2}=\frac{x}{L}, \quad t=a x^{+}, \\
\phi_{1} & =b x^{+}+\frac{x^{-}}{L^{2}}, \quad \phi_{2} \rightarrow \frac{\phi_{2}}{L}, \quad \psi \rightarrow \frac{\psi}{L} .
\end{aligned}
$$

The null geodesic condition gives

$$
a^{2}=\frac{2 \lambda_{1}^{2} b^{2}}{c^{2}} \ln \left(\frac{c}{\tilde{r}}\right)
$$

The leading terms of the $T$-dual metric in the limit $L \rightarrow \infty$ are given by

$$
\begin{aligned}
d s^{2}= & 2 \sqrt{2} \lambda_{1}^{2} b \sqrt{\ln \left(\frac{c}{\tilde{r}}\right)} d x^{+} d x^{-}+\frac{1}{\sqrt{2}} \frac{c^{2}}{\sqrt{\ln \left(\frac{c}{\tilde{r}}\right)}}\left(d y_{1}^{2}+d y_{2}^{2}+d y_{3}^{2}\right)+\frac{\sqrt{2}}{c^{2}} \sqrt{\ln \left(\frac{c}{\tilde{r}}\right)} d w^{2} \\
& +\lambda_{1}^{2} \sqrt{2} \sqrt{\ln \left(\frac{c}{\tilde{r}}\right)} d z^{2}+\lambda_{2}^{2} \sqrt{2} \sqrt{\ln \left(\frac{c}{\tilde{r}}\right)} d x^{2}+\lambda^{2} \sqrt{2} \sqrt{\ln \left(\frac{c}{\tilde{r}}\right)}\left(\left(d \psi+d \phi_{2}\right)^{2}\right. \\
& \left.-2 b z d x^{+} d \psi-2 b z d x^{+} d \phi_{2}\right)+\left[\frac{1}{\sqrt{2}} \frac{-w^{2} a^{2}}{\sqrt{\ln \left(\frac{c}{\tilde{r}}\right)}}\left(\frac{1}{4 \ln \left(\frac{c}{\tilde{r}}\right)}+1\right)+\sqrt{2} \sqrt{\ln \left(\frac{c}{\tilde{r}}\right)} b^{2} z^{2}\left(\lambda^{2}-\lambda_{1}^{2}\right)\right. \\
& \left.-\frac{w^{2} b^{2}}{4 c^{2} \ln \left(\frac{c}{\tilde{r}}\right)}\right]\left(d x^{+}\right)^{2}+L\left[\frac{1}{\sqrt{2}} \frac{c w a^{2}}{\sqrt{\ln \left(\frac{c}{\tilde{r}}\right)}}\left(\frac{1}{2 \ln \left(\frac{c}{\tilde{r}}\right)}-2\right)+\frac{\lambda_{1}^{2}}{c \sqrt{2}} \frac{w b^{2}}{\sqrt{\ln \left(\frac{c}{\tilde{r}}\right)}}\right]\left(d x^{+}\right)^{2} .
\end{aligned}
$$

Once again, the metric is divergent in the limit $L \rightarrow \infty$ due to the presence of the $\mathcal{O}(L)$ term.

We will now consider a null geodesic which carries angular momentum. To obtain such a geodesic, we consider motion along $r$ and $\phi_{1}$ directions and concentrate in a small neighborhood of $\theta_{1}=\theta_{2}=\phi_{2}=\psi=0$. The Lagrangian for a massless particle moving along this geodesic is

$$
\mathcal{L}=\frac{1}{2} g_{\mu \nu} \dot{X}^{\mu} \dot{X}^{\nu}
$$

Here dots denote derivative with respect to the affine parameter $u$. Substituting the explicit expression for the background metric (A4) in the above Lagrangian we find

$$
\begin{aligned}
\mathcal{L}= & \frac{L^{2}}{2}\left(-\frac{1}{\sqrt{2}} \frac{r^{2}}{\sqrt{\ln \left(\frac{r}{\tilde{r}}\right)}} \dot{t}^{2}+\frac{\sqrt{2}}{r^{2}} \sqrt{\ln \left(\frac{r}{\tilde{r}}\right)} \dot{r}^{2}\right. \\
& \left.+\lambda^{2} \sqrt{2} \sqrt{\ln \left(\frac{r}{\tilde{r}}\right)} \dot{\phi}_{1}^{2}\right) .
\end{aligned}
$$

We notice that the above Lagrangian does not depend on $t$ and $\phi_{1}$ explicitly. Hence the momenta conjugate to the generalized coordinates $t$ and $\phi_{1}$ are conserved. Denoting these quantities by $E$ and $J$ (up to a factor of $-L^{2}$ ), we find

$$
\begin{gathered}
\frac{\partial \mathcal{L}}{\partial \dot{t}}=-\frac{L^{2}}{\sqrt{2}} \frac{r^{2}}{\sqrt{\ln \left(\frac{r}{\tilde{r}}\right)}} \dot{t}=-E L^{2}, \\
\frac{\partial \mathcal{L}}{\partial \dot{\phi}_{1}}=\lambda^{2} L^{2} \sqrt{2} \sqrt{\ln \left(\frac{r}{\tilde{r}}\right)} \dot{\phi}_{1}=-J L^{2} .
\end{gathered}
$$

The condition that the geodesic becomes null gives rise to

$$
\dot{r}^{2}+\frac{J^{2} r^{2}}{2 \lambda^{2} \ln \left(\frac{r}{\tilde{r}}\right)}=E^{2} .
$$

To obtain the Penrose limit, we redefine the coordinates as

$$
\begin{aligned}
x_{i} & =\frac{y_{i}}{L} ; \quad i=1,2,3, \quad \theta_{1}=\frac{z}{L}, \quad \theta_{2}=\frac{x}{L}, \\
\phi_{2} & \rightarrow \frac{\phi_{2}}{L}, \quad \psi \rightarrow \frac{\psi}{L},
\end{aligned}
$$

and consider the following expansion in the limit $L \rightarrow \infty$ :

$$
\begin{aligned}
d t & =c_{1} d u, \quad d r=c_{2} d u+c_{3} \frac{d w}{L}, \\
d \phi_{1} & =c_{4} d u+c_{5} \frac{d w}{L}+c_{6} \frac{d v}{L^{2}} .
\end{aligned}
$$

By requiring the geodesic to be null determines the coefficients $c_{1}, c_{2}$, and $c_{4}$ as 


$$
c_{1}=\frac{E \sqrt{2}}{r^{2}} \sqrt{\ln \left(\frac{r}{\tilde{r}}\right)}, \quad c_{2}=\left[E^{2}-\frac{J^{2} r^{2}}{2 \lambda^{2} \ln \left(\frac{r}{\tilde{r}}\right)}\right]^{\frac{1}{2}}, \quad c_{4}=-\frac{J}{\lambda^{2} \sqrt{2} \sqrt{\ln \left(\frac{r}{\tilde{r}}\right)}} .
$$

The metric then becomes

$$
\begin{aligned}
d s^{2}= & 2 \sqrt{2} \lambda^{2} \sqrt{\ln \left(\frac{r}{\tilde{r}}\right)} c_{4} c_{6} d u d v+\frac{1}{\sqrt{2}} \frac{r^{2}}{\sqrt{\ln \left(\frac{r}{\tilde{r}}\right)}}\left(d y_{1}^{2}+d y_{2}^{2}+d y_{3}^{2}\right) \\
& +\sqrt{2} \sqrt{\ln \left(\frac{r}{\tilde{r}}\right)}\left(\frac{c_{3}^{2}}{r^{2}}+\lambda^{2} c_{5}^{2}\right) d w^{2}+\lambda_{1}^{2} \sqrt{2} \sqrt{\ln \left(\frac{r}{\tilde{r}}\right)} d z^{2}+\lambda_{2}^{2} \sqrt{2} \sqrt{\ln \left(\frac{r}{\tilde{r}}\right)} d x^{2} \\
& +\lambda^{2} \sqrt{2} \sqrt{\ln \left(\frac{r}{\tilde{r}}\right)}\left(\left(d \psi+d \phi_{2}\right)^{2}+2 c_{3} d w\left(d \psi+d \phi_{2}\right)\right)+\sqrt{2} \sqrt{\ln \left(\frac{r}{\tilde{r}}\right)} c_{4}^{2} z^{2}\left(\lambda_{1}^{2}-\lambda^{2}\right) d u^{2} \\
& +L\left[\frac{2 \sqrt{2}}{r^{2}} \sqrt{\ln \left(\frac{r}{\tilde{r}}\right)}\left(c_{2} c_{3}+\lambda^{2} r^{2} c_{4} c_{5}\right) d u d w+2 c_{4}\left(d \psi+d \phi_{2}\right) d u\right] .
\end{aligned}
$$

We note that the divergent term of order $\mathcal{O}(L)$ cannot be removed. Thus the Penrose limit around this geodesic does not give a smooth geometry.

Finally, we will consider the expansion around $\theta_{1}=\frac{\pi}{2}$ and concentrate in a small neighborhood of $\theta_{2}=\phi_{2}=\psi=0$. The Lagrangian for a massless particle then gives rise to

$$
\mathcal{L}=\frac{L^{2}}{2}\left(-\frac{1}{\sqrt{2}} \frac{r^{2}}{\sqrt{\ln \left(\frac{r}{\tilde{r}}\right)}} \dot{t}^{2}+\frac{\sqrt{2}}{r^{2}} \sqrt{\ln \left(\frac{r}{\tilde{r}}\right)} \dot{r}^{2}+\lambda_{1}^{2} \sqrt{2} \sqrt{\ln \left(\frac{r}{\tilde{r}}\right)} \dot{\phi}_{1}^{2}\right) .
$$

The conserved quantities are

$$
\begin{aligned}
& \frac{\partial \mathcal{L}}{\partial \dot{t}}=-\frac{L^{2}}{\sqrt{2}} \frac{r^{2}}{\sqrt{\ln \left(\frac{r}{\tilde{r}}\right)}} \dot{t}=-E L^{2}, \\
& \frac{\partial \mathcal{L}}{\partial \dot{\phi}_{1}}=\lambda_{1}^{2} L^{2} \sqrt{2} \sqrt{\ln \left(\frac{r}{\tilde{r}}\right)} \dot{\phi}_{1}=-J L^{2},
\end{aligned}
$$

and the null geodesic condition becomes

$$
\dot{r}^{2}+\frac{J^{2} r^{2}}{2 \lambda_{1}^{2} \ln \left(\frac{r}{\tilde{r}}\right)}=E^{2}
$$

To obtain the Penrose limit, we redefine the coordinates

$$
x_{i}=\frac{y_{i}}{L} ; \quad i=1,2,3, \quad \theta_{1}=\frac{\pi}{2}+\frac{z}{L}, \quad \theta_{2}=\frac{x}{L}, \quad \phi_{2} \rightarrow \frac{\phi_{2}}{L}, \quad \psi \rightarrow \frac{\psi}{L},
$$

Then consider the following expansion in the limit $L \rightarrow \infty$ :

$$
d t=c_{1} d u, \quad d r=c_{2} d u+c_{3} \frac{d w}{L}, \quad d \phi_{1}=c_{4} d u+c_{5} \frac{d w}{L}+c_{6} \frac{d v}{L^{2}} .
$$

The null geodesic condition determines the coefficients $c_{1}, c_{2}$, and $c_{4}$ as

$$
c_{1}=\frac{E \sqrt{2}}{r^{2}} \sqrt{\ln \left(\frac{r}{\tilde{r}}\right)}, \quad c_{2}=\left[E^{2}-\frac{J^{2} r^{2}}{2 \lambda^{2} \ln \left(\frac{r}{\tilde{r}}\right)}\right]^{\frac{1}{2}}, \quad c_{4}=-\frac{J}{\lambda_{1}^{2} \sqrt{2} \sqrt{\ln \left(\frac{r}{\tilde{r}}\right)}} .
$$

The background metric then takes the form 


$$
\begin{aligned}
d s^{2}= & 2 \sqrt{2} \lambda_{1}^{2} \sqrt{\ln \left(\frac{r}{\tilde{r}}\right)} c_{4} c_{6} d u d v+\frac{1}{\sqrt{2}} \frac{r^{2}}{\sqrt{\ln \left(\frac{r}{\tilde{r}}\right)}}\left(d y_{1}^{2}+d y_{2}^{2}+d y_{3}^{2}\right) \\
& +\sqrt{2} \sqrt{\ln \left(\frac{r}{\tilde{r}}\right)}\left(\frac{c_{3}^{2}}{r^{2}}+\lambda_{1}^{2} c_{5}^{2}\right) d w^{2}+\lambda_{1}^{2} \sqrt{2} \sqrt{\ln \left(\frac{r}{\tilde{r}}\right)} d z^{2}+\lambda_{2}^{2} \sqrt{2} \sqrt{\ln \left(\frac{r}{\tilde{r}}\right)} d x^{2} \\
& +\lambda^{2} \sqrt{2} \sqrt{\ln \left(\frac{r}{\tilde{r}}\right)}\left(\left(d \psi+d \phi_{2}\right)^{2}-2 c_{4} z d u\left(d \psi+d \phi_{2}\right)\right)+\sqrt{2} \sqrt{\ln \left(\frac{r}{\tilde{r}}\right)} c_{4}^{2} z^{2}\left(\lambda^{2}-\lambda_{1}^{2}\right) d u^{2}
\end{aligned}
$$

along with an order $\mathcal{O}(L)$ divergent term which can be removed upon requiring

$$
c_{2} c_{3}+\lambda_{1}^{2} r^{2} c_{4} c_{5}=0
$$

thereby giving rise to a smooth geometry. However, a straightforward calculation gives rise to a nonvanishing scalar curvature for this geometry. Hence, this does not correspond to a pp-wave.

\section{APPENDIX B: EINSTEIN'S EQUATIONS}

In this Appendix, we will analyze Einstein's equations for our pp-wave background. Einstein's equation for type-IIA supergravity is given by

$$
\hat{R}_{\mu \nu}+2 D_{\mu} D_{\nu} \hat{\Phi}=\frac{1}{4} \hat{H}_{\mu \nu}^{2}+e^{2 \hat{\Phi}}\left[\frac{1}{2}\left(\hat{F}_{2}^{2}\right)_{\mu \nu}+\frac{1}{12}\left(\hat{F}_{4}^{2}\right)_{\mu \nu}-\frac{1}{4} g_{\mu \nu}\left(\hat{F}_{0}^{2}+\frac{1}{2} \hat{F}_{2}^{2}+\frac{1}{4 !} \hat{F}_{4}^{2}\right)\right]
$$

Here we use the conventions of [15]. In particular, we have $\hat{H}_{\mu \nu}^{2}=\hat{H}_{\mu \alpha \beta} \hat{H}_{\nu \rho \sigma} g^{\alpha \rho} g^{\beta \sigma}$ and similar expressions for $\left(\hat{F}_{2}^{2}\right)_{\mu \nu}$ and $\left(\hat{F}_{4}^{2}\right)_{\mu \nu}$. The equation of motion for the dilation is given by

$$
\hat{R}+4 D^{2} \hat{\Phi}-4(\partial \hat{\Phi})^{2}-\frac{1}{12} \hat{H}^{2}=0
$$

We will first focus on the dilation equation. For the pp-wave background $\hat{R}=0$. Now, consider evaluating $D^{2} \hat{\Phi}$. Note that

$$
D^{2} \hat{\Phi}=g^{\mu \nu} D_{\mu} D_{\nu} \hat{\Phi}=g^{u v} D_{u} D_{v} \hat{\Phi}+g^{v u} D_{v} D_{u} \hat{\Phi}+g^{v v} D_{v} D_{v} \hat{\Phi}+g^{i j} D_{i} D_{j} \hat{\Phi}
$$

for $i, j \neq\{u, v\}$. Consider the covariant derivatives of the form $D_{\mu} D_{\nu} \hat{\Phi}$ appearing in the above equation. Since $\partial_{v} \hat{\Phi}=0=\partial_{i} \hat{\Phi}$, we find

$$
\begin{aligned}
& D_{u} D_{v} \hat{\Phi}=\partial_{u} \partial_{v} \hat{\Phi}-\Gamma_{u v}^{\lambda} \partial_{\lambda} \hat{\Phi}=-\Gamma_{u v}^{u} \partial_{u} \hat{\Phi} \\
& D_{v} D_{u} \hat{\Phi}=\partial_{v} \partial_{u} \hat{\Phi}-\Gamma_{v u}^{\lambda} \partial_{\lambda} \hat{\Phi}=-\Gamma_{v u}^{u} \partial_{u} \hat{\Phi} \\
& D_{v} D_{v} \hat{\Phi}=\partial_{v} \partial_{v} \hat{\Phi}-\Gamma_{v v}^{\lambda} \partial_{\lambda} \hat{\Phi}=-\Gamma_{v v}^{u} \partial_{u} \hat{\Phi} \\
& D_{i} D_{j} \hat{\Phi}=\partial_{i} \partial_{j} \hat{\Phi}-\Gamma_{i j}^{\lambda} \partial_{\lambda} \hat{\Phi}=-\Gamma_{i j}^{u} \partial_{u} \hat{\Phi} .
\end{aligned}
$$

It is straightforward to evaluate the Christoffel symbols. We find

$$
\begin{gathered}
\Gamma_{u v}^{u}=\frac{1}{2} g^{u v}\left(\partial_{v} g_{v u}+\partial_{u} g_{v v}-\partial_{v} g_{u v}\right)=0, \\
\Gamma_{v v}^{u}=\frac{1}{2} g^{u v}\left(\partial_{v} g_{v v}+\partial_{v} g_{v v}-\partial_{v} g_{u v}\right)=0, \\
\Gamma_{i j}^{u}=\frac{1}{2} g^{u v}\left(\partial_{j} g_{v i}+\partial_{i} g_{v j}-\partial_{v} g_{i j}\right)=0 .
\end{gathered}
$$

Thus, we have $D^{2} \hat{\Phi}=0$. Similarly, we can show that $(\partial \hat{\Phi})^{2}$ also vanishes identically: 


$$
(\partial \hat{\Phi})^{2}=g^{\mu \nu} \partial_{\mu} \hat{\Phi} \partial_{\nu} \hat{\Phi}=2 g^{u v} \partial_{u} \hat{\Phi} \partial_{v} \hat{\Phi}+g^{v v} \partial_{v} \hat{\Phi} \partial_{v} \hat{\Phi}+g^{i j} \partial_{i} \hat{\Phi} \partial_{j} \hat{\Phi}=0 .
$$

Further, from the expression for $\hat{H}_{3}$, we find that $\hat{H}_{3}^{2}=0$. This shows that the dilaton equation is satisfied identically.

We will now consider Einstein's equations. Clearly, from (3.41) we have $\hat{F}_{0}^{2}=\hat{F}_{2}^{2}=\hat{F}_{4}^{2}=0$. Further, a straightforward calculation shows that the only the $u u$ components of $\hat{H}_{\mu \nu}^{2},\left(\hat{F}_{2}\right)_{\mu \nu}^{2},\left(\hat{F}_{4}\right)_{\mu \nu}^{2}$ together with $D_{u} D_{u} \hat{\Phi}$ are nonvanishing. Likewise, we know from [5] that in Brinkmann coordinates the $u u$ component $\hat{R}_{u u}$ is the only nonvanishing component of the Ricci tensor. Thus, for our background Einstein's equation reduces to

$$
\hat{R}_{u u}+2 D_{u} D_{u} \hat{\Phi}=\frac{1}{4} \hat{H}_{u u}^{2}+e^{2 \hat{\Phi}}\left[\frac{1}{2}\left(\hat{F}_{2}^{2}\right)_{u u}+\frac{1}{12}\left(\hat{F}_{4}^{2}\right)_{u u}\right] .
$$

In the following we evaluate each of the terms of the above equation. The nonzero component of the Ricci tensor is

$$
\hat{R}_{u u}=-\left[F_{y_{1}}+F_{y_{2}}+F_{y_{3}}+F_{w}+\left(F_{z}-\frac{3 \sqrt{2}}{\sqrt{\ln \left(\frac{r}{\tilde{r}}\right)}} J^{2}\right)+F_{v_{2}}+F_{v_{3}}\right],
$$

where the expressions for the functions $F_{i}$ are given in (3.36). Similarly, the remaining terms in the equation are evaluated to be

$$
\begin{aligned}
e^{-2 \hat{\Phi}}= & \frac{4 \sqrt{2}}{81 \tilde{g}_{s}^{2}} \sqrt{\ln \left(\frac{r}{\tilde{r}}\right)}\left[\ln \left(\frac{r}{\tilde{r}}\right)+2\left(\ln \left(\frac{r}{r_{0}}\right)\right)^{2}\right], \\
D_{u} D_{u} \hat{\Phi}= & \frac{1}{8 r^{2}\left(\ln \left(\frac{r}{\tilde{r}}\right)\right)^{3}\left[\ln \left(\frac{r}{\tilde{r}}\right)+2\left(\ln \left(\frac{r}{r_{0}}\right)\right)^{2}\right]^{2}} f(r), \\
\hat{H}_{u u}^{2}= & 2\left[\frac{\sqrt{2}}{3}\left(E^{2}-\frac{3 r^{2}}{\ln \left(\frac{r}{\tilde{r}}\right)} J^{2}\right)\left(c_{5}^{\prime} \ln \left(\frac{r}{r_{0}}\right)+\frac{c_{5}}{r}\right)^{2}\left(\frac{c_{3}^{2} \sqrt{2}}{r^{2}}+\frac{c_{5}^{2}}{3 \sqrt{2}}\right)^{-1} \frac{1}{\ln \left(\frac{r}{\tilde{r}}\right)}\right. \\
& +2\left(E^{2}-\frac{3 r^{2}}{\ln \left(\frac{r}{\tilde{r}}\right)} J^{2}\right)\left(\frac{\ln \left(\frac{r}{\tilde{r}}\right)-\ln \left(\frac{r}{r_{0}}\right)-2\left(\ln \left(\frac{r}{r_{0}}\right)\right)^{2}}{r\left(\ln \left(\frac{r}{\tilde{r}}\right)+2\left(\ln \left(\frac{r}{r_{0}}\right)\right)^{2}\right)} \frac{1}{\ln \left(\frac{r}{\tilde{r}}\right)}+\frac{12 J^{2}}{\ln \left(\frac{r}{\tilde{r}}\right)}\right], \\
\left(\hat{F}_{2}\right)_{u u}^{2}= & \frac{2^{\left(-\frac{3}{2}\right)}}{243 \tilde{g}_{s}^{2}} \frac{J^{2}}{\left(\ln \left(\frac{r}{\tilde{r}}\right)\right)^{\frac{3}{2}}}\left[\frac{2}{5} \ln \left(\frac{r}{\tilde{r}}\right)+24 \ln \left(\frac{r}{r_{0}}\right)-\frac{1}{10}\right]^{2}, \\
\left(\hat{F}_{4}\right)_{u u}^{2}= & \frac{2^{\frac{9}{2}}}{9 \tilde{g}_{s}^{2}} \frac{J^{2}}{\left(\ln \left(\frac{r}{\tilde{r}}\right)\right)^{\frac{5}{2}}}\left[-\ln \left(\frac{r}{\tilde{r}}\right)+\frac{1}{2} \ln \left(\frac{r}{r_{0}}\right)\left(\frac{1}{15} \ln \left(\frac{r}{\tilde{r}}\right)-\frac{1}{60}\right)\right]^{2} .
\end{aligned}
$$

where the function $f(r)$ has the complicated expression:

$$
\begin{aligned}
f(r)= & -36 J^{2} r^{2}\left(\ln \left(\frac{r}{r_{0}}\right)\right)^{4}+2 E^{2}\left(\ln \left(\frac{r}{\tilde{r}}\right)\right)^{4}\left(-5+8 \ln \left(\frac{r}{r_{0}}\right)\right) \\
& +8 \ln \left(\frac{r}{\tilde{r}}\right)\left(\ln \left(\frac{r}{r_{0}}\right)\right)^{2}\left(-6 J^{2} r^{2}-6 J^{2} r^{2} \ln \left(\frac{r}{r_{0}}\right)+E^{2}\left(\ln \left(\frac{r}{r_{0}}\right)\right)^{2}\right) \\
& +\left(\ln \left(\frac{r}{\tilde{r}}\right)\right)^{3}\left(6\left(E^{2}+8 J^{2} r^{2}\right)+32 E^{2} \ln \left(\frac{r}{r_{0}}\right)+48 E^{2}\left(\ln \left(\frac{r}{r_{0}}\right)\right)^{2}+32 E^{2}\left(\ln \left(\frac{r}{r_{0}}\right)\right)^{3}\right) \\
& +\left(\ln \left(\frac{r}{\tilde{r}}\right)\right)^{2}\left(-27 J^{2} r^{2}-120 J^{2} r^{2} \ln \left(\frac{r}{r_{0}}\right)+8\left(E^{2}-12 J^{2} r^{2}\right)\left(\ln \left(\frac{r}{r_{0}}\right)\right)^{2}+8 E^{2}\left(\ln \left(\frac{r}{r_{0}}\right)\right)^{4}\right) .
\end{aligned}
$$

Substituting the above expressions in (B7) we find that it does not vanish identically. Since it involves the only unknown quantity $c_{5}$, we can use this equation to determine it. Though it is possible to reproduce the exact expression for $c_{5}$ in closed form, it is rather complicated and unimaginative. Thus we skip writing it here. 
[1] J. Kowalski-Glikman, Vacuum states in supersymmetric Kaluza-Klein theory, Phys. Lett. 134B, 194 (1984).

[2] P. T. Chrusciel and J. Kowalski-Glikman, The isometry group and killing spinors for the pp wave space-time in $D=11$ supergravity, Phys. Lett. 149B, 107 (1984).

[3] J. M. Figueroa-O'Farrill and G. Papadopoulos, Homogeneous fluxes, branes and a maximally supersymmetric solution of M theory, J. High Energy Phys. 08 (2001) 036.

[4] M. Blau, J. M. Figueroa-O'Farrill, C. Hull, and G. Papadopoulos, A new maximally supersymmetric background of IIB superstring theory, J. High Energy Phys. 01 (2002) 047.

[5] M. Blau, Plane waves and Penrose limits, http://www.blau .itp.unibe.ch/lecturesPP.pdf.

[6] M. Blau, J. M. Figueroa-O'Farrill, C. Hull, and G. Papadopoulos, Penrose limits and maximal supersymmetry, Classical Quant. Grav. 19, L87 (2002).

[7] D. E. Berenstein, J. M. Maldacena, and H. S. Nastase, Strings in flat space and pp waves from $\mathcal{N}=4$ superYang-Mills, J. High Energy Phys. 04 (2002) 013.

[8] D. Sadri and M. M. Sheikh-Jabbari, The plane wave/superYang-Mills duality, Rev. Mod. Phys. 76, 853 (2004).

[9] D. Amati and C. Klimcik, Nonperturbative computation of the Weyl anomaly for a class of nontrivial backgrounds, Phys. Lett. B 219, 443 (1989).

[10] G. T. Horowitz and A. R. Steif, Space-Time Singularities in String Theory, Phys. Rev. Lett. 64, 260 (1990).

[11] X. C. de la Ossa and F. Quevedo, Duality symmetries from non-Abelian isometries in string theory, Nucl. Phys. B403, 377 (1993).

[12] A. Giveon and M. Rocek, On non-Abelian duality, Nucl. Phys. B421, 173 (1994).

[13] K. Sfetsos and D.C. Thompson, On non-Abelian T-dual geometries with Ramond fluxes, Nucl. Phys. B846, 21 (2011).

[14] Y. Lozano, E. O Colgain, K. Sfetsos, and D. C. Thompson, Non-Abelian $T$-duality, Ramond fields and coset geometries, J. High Energy Phys. 06 (2011) 106.

[15] G. Itsios, Y. Lozano, E. O Colgain, and K. Sfetsos, NonAbelian $T$-duality and consistent truncations in type-II supergravity, J. High Energy Phys. 08 (2012) 132.

[16] G. Itsios, C. Nunez, K. Sfetsos, and D. C. Thompson, On non-Abelian $T$-duality and new $\mathcal{N}=1$ backgrounds, Phys. Lett. B 721, 342 (2013).

[17] A. Barranco, J. Gaillard, N. T. Macpherson, C. Nunez, and D. C. Thompson, G-structures and flavouring non-Abelian T-duality, J. High Energy Phys. 08 (2013) 018.

[18] N. T. Macpherson, C. Núñez, L. A. Pando Zayas, V. G. J. Rodgers, and C. A. Whiting, Type IIB supergravity solutions with $\mathrm{AdS}_{5}$ from Abelian and non-Abelian T dualities, J. High Energy Phys. 02 (2015) 040.

[19] K. S. Kooner and S. Zacarias, Non-Abelian T-dualizing the resolved conifold with regular and fractional D3-branes, J. High Energy Phys. 08 (2015) 143.

[20] H. Dimov, S. Mladenov, R. C. Rashkov, and T. Vetsov, NonAbelian $T$-duality of Pilch-Warner background, Fortschr. Phys. 64, 657 (2016).

[21] H. Dimov, R. C. Rashkov, S. Mladenov, and T. Vetsov, NonAbelian $T$-duality from penrose limit of the Pilch-Warner solution, Bulg. J. Phys. 43, 251 (2016), http://www.bjp-bg .com/papers/bjp2016_4_251-265.pdf.
[22] Y. Lozano and C. Nuenz, Field theory aspects of nonAbelian $T$-duality and $\mathcal{N}=2$ linear quivers, J. High Energy Phys. 05 (2016) 107.

[23] Y. Lozano, N. T. Macpherson, J. Montero, and C. Nunez, Three-dimensional $\mathcal{N}=4$ linear quivers and non-Abelian T-duals, J. High Energy Phys. 11 (2016) 133.

[24] G. Itsios, C. Nunez, K. Sfetsos, and D. C. Thompson, NonAbelian $T$-duality and the AdS/CFT correspondence: New $\mathcal{N}=1$ backgrounds, Nucl. Phys. B873, 1 (2013).

[25] T. R. Araujo and H. Nastase, $\mathcal{N}=1$ SUSY backgrounds with an AdS factor from non-Abelian $T$ duality, Phys. Rev. D 91, 126015 (2015).

[26] C. A. Whiting, Duality symmetries in string-inspired supergravity: $T$-dualities and the gauge/gravity correspondence, Ph.D. Thesis, University of Iowa, 2015, 10.17077/ etd.qc6n8jma.

[27] G. Itsios, H. Nastase, C. Nez, K. Sfetsos, and S. Zacaras, Penrose limits of Abelian and non-Abelian $T$-duals of $\mathrm{AdS}_{5} \times S^{5}$ and their field theory duals, J. High Energy Phys. 01 (2018) 071.

[28] G. Itsios, Y. Lozano, J. Montero, and C. Nunez, The $\mathrm{AdS}_{5}$ non-Abelian $T$-dual of Klebanov-Witten as a $\mathcal{N}=1$ linear quiver from M5-branes, J. High Energy Phys. 09 (2017) 038 .

[29] J. Gaillard, N. T. Macpherson, C. Núñez, and D. C. Thompson, Dualising the baryonic branch: Dynamic SU(2) and confining backgrounds in IIA, Nucl. Phys. B884, 696 (2014).

[30] R. Penrose, Any space-time has a plane wave as a limit, in Differential Geometry and Relativity (Springer, Dordrecht, 1976), pp. 271-275.

[31] I. R. Klebanov and E. Witten, Superconformal field theory on three-branes at a Calabi-Yau singularity, Nucl. Phys. B536, 199 (1998).

[32] I. R. Klebanov and E. Witten, AdS/CFT correspondence and symmetry breaking, Nucl. Phys. B556, 89 (1999).

[33] S. Roychowdhury and P. K. Tripathy, The non-Abelian $T$-dual of Klebanov-Witten background and its Penrose limits, J. High Energy Phys. 11 (2019) 125.

[34] I. R. Klebanov and A. A. Tseytlin, Gravity duals of supersymmetric $S U(N) \times S U(N+M)$ gauge theories, Nucl. Phys. B578, 123 (2000).

[35] M. Kruczenski, Wilson loops and anomalous dimensions in cascading theories, Phys. Rev. D 69, 106002 (2004).

[36] L. A. Pando Zayas and J. Sonnenschein, On Penrose limits and gauge theories, J. High Energy Phys. 05 (2002) 010.

[37] S. F. Hassan, T duality, space-time spinors and RR fields in curved backgrounds, Nucl. Phys. B568, 145 (2000).

[38] J. Jeong, O. Kelekci, and E. O Colgain, An alternative IIB embedding of F(4) gauged supergravity, J. High Energy Phys. 05 (2013) 079.

[39] O. Kelekci, Y. Lozano, N. T. Macpherson, and E. O. Colgain, Supersymmetry and non-Abelian $T$-duality in type II supergravity, Classical Quant. Grav. 32, 035014 (2015).

[40] I. R. Klebanov and M. J. Strassler, Supergravity and a confining gauge theory: Duality cascades and $\chi$ SBresolution of naked singularities, J. High Energy Phys. 08 (2000) 052. 